\title{
A.E. CONVERGENCE AND 2-WEIGHT INEQUALITIES FOR POISSON-LAGUERRE SEMIGROUPS
}

\author{
G. GARRIGÓS, S.HARTZSTEIN, T. SIGNES, B. VIVIANI
}

\begin{abstract}
We find optimal decay estimates for the Poisson kernels associated with various Laguerre-type operators $L$. From these, we solve two problems about the Poisson semigroup $e^{-t \sqrt{L}}$. First, we find the largest space of initial data $f$ so that $e^{-t \sqrt{L}} f(x) \rightarrow f(x)$ at a.e. $x$. Secondly, we characterize the largest class of weights $w$ which admit 2 -weight inequalities of the form $\left\|\sup _{0<t \leq t_{0}}\left|e^{-t \sqrt{L}} f\right|\right\|_{L^{p}(v)} \lesssim\|f\|_{L^{p}(w)}$, for some other weight $v$.
\end{abstract}

\section{INTRODUCTION}

In this paper we continue the research, started in [6, 5], about Poisson integrals associated with certain differential operators $L$, say symmetric and positive in $L^{2}(\varnothing, \mu)$. Namely, we are interested in the behavior of

$$
u(t, x)=e^{-t \sqrt{L}} f(x)
$$

as a solution of the elliptic differential equation

$$
\left\{\begin{array}{l}
u_{t t}-L u=0 \\
u(0, x)=f(x),
\end{array} \text { in the half-plane }(0, \infty) \times \varnothing .\right.
$$

We shall study two questions, which are closely related among themselves

(i) find the largest class of functions $f$ for which $\lim _{t \rightarrow 0^{+}} u(t, x)=f(x)$, a.e. $x \in \emptyset$;

(ii) establish 2-weight inequalities of the form

$$
\left\|\sup _{0<t \leq t_{0}}|u(t, x)|\right\|_{L^{p}(v)} \lesssim\|f\|_{L^{p}(w)}
$$

for the largest class of weights $w$ for which a suitable $v$ with this property exists.

In [5] we considered these questions in full detail when $L$ is the Hermite operator. In this paper we intend to do the same for the various Laguerre operators. We remark that solving these questions for the largest class of weights or initial data is generally not an easy task, requiring optimal decay estimates of the Poisson kernels. These new estimates have an independent interest and may be useful in other settings; see e.g. recent work by Liu and Sjögren [7]. For our purposes, they will provide a.e. convergence for new initial data $f$ compared to Muckenhoupt [8] and Stempak [12], and also for larger weight classes compared to those of Nowak in [9].

Date: July 2, 2019.

2010 Mathematics Subject Classification. 33C45, 35C15, 40A10, 42C10, 47D06.

Key words and phrases. Laguerre expansions, Poisson integral, heat semigroup, weights. 
We now state our results, for simplicity in the special case of the classical Laguerre operator in $\mathbb{R}_{+}:=(0, \infty)$

$$
\mathbb{L}=-y \partial_{y y}-(\alpha+1-y) \partial_{y}+m, \quad \text { where } \alpha>-1 \quad \text { and } \quad m \geq 0 .
$$

We have incorporated a parameter $m \geq 0$ which later will allow us to recover other Laguerretype operators (after suitable changes of variables). We shall also consider a slightly more general family of partial differential equations, namely

$$
\left\{\begin{array}{l}
u_{t t}+\frac{1-2 \nu}{t} u_{t}=\mathbb{L} u \\
u(t, 0)=f,
\end{array} \quad \text { where } \nu>0 .\right.
$$

These pde's appear in relation with the fractional operator $f \mapsto \mathbb{L}^{\nu} f$ (see e.g. [14]).

As discussed in [14, a candidate solution to (1.2) is given by the Poisson-like integral

$$
P_{t} f(x):=\frac{t^{2 \nu}}{4^{\nu} \Gamma(\nu)} \int_{0}^{\infty} e^{-\frac{t^{2}}{4 u}}\left[e^{-u \mathbb{L}} f\right](x) \frac{d u}{u^{1+\nu}}, \quad t>0,
$$

which is subordinated to the "heat" semigroup $\left\{e^{-u \mathbb{L}}\right\}_{u>0}$. Our first goal is to find the most general conditions on a function $f: \mathbb{R}_{+} \rightarrow \mathbb{C}$ so that $P_{t} f$ is a meaningful solution of (1.2). These conditions will depend on the following weight

$$
\Phi(y)=\frac{y^{\alpha} e^{-y}}{(1+y)^{m}[\ln (e+y)]^{1+\nu}} \quad \text { if } m>0, \quad \text { and } \quad \Phi(y)=\frac{y^{\alpha} e^{-y}}{[\ln (e+y)]^{\nu}} \text { if } m=0 .
$$

Theorem 1.1. For every $f \in L^{1}(\Phi)$ the function $u(t, x)=P_{t} f(x)$ in (1.3) is defined by an absolutely convergent integral such that

(i) $u(t, x) \in C^{\infty}\left((0, \infty) \times \mathbb{R}_{+}\right)$and satisfies the pde (1.2)

(ii) $\lim _{t \rightarrow 0^{+}} u(t, x)=f(x)$ at a.e. $x \in \mathbb{R}_{+}$.

Conversely, if a function $f \geq 0$ is such that the integral in (1.3) is finite for some $(t, x) \in$ $(0, \infty) \times \mathbb{R}_{+}$, then $f$ must necessarily belong to $L^{1}(\Phi)$.

We recall that Muckenhoupt proved in $[8]$ the pointwise convergence for data $f$ in the smaller space $L^{1}\left(y^{\alpha} e^{-y} d y\right.$ ) (in the classical setting, i.e., (1.1) with $m=0$ and (1.3) with $\nu=1 / 2$ ). Our result, which is sharp on positive $f$, enlarges the space to

$$
L^{1}\left(y^{\alpha} e^{-y} / \sqrt{\log (e+y)} d y\right),
$$

and allows to include new initial data such as $f(y)=e^{y} /\left[(1+y)^{\alpha+1} \log (e+y)\right]$.

Our second question concerns more "quantitative" bounds for the solutions of (1.2), expressed in terms of the following local maximal operators

$$
P_{t_{0}}^{*} f(x):=\sup _{0<t \leq t_{0}}\left|P_{t} f(x)\right|, \quad \text { with } t_{0}>0 \text { fixed. }
$$


From our estimates of the Poisson kernels we shall be able to prove that

$$
P_{t_{0}}^{*}: L^{1}(\Phi) \rightarrow L_{\mathrm{loc}}^{s} \quad \text { if } s<1, \quad \text { and } \quad P_{t_{0}}^{*}: L^{1}(\Phi) \cap L_{\mathrm{loc}}^{p} \rightarrow L_{\mathrm{loc}}^{p} \quad \text { if } p>1 .
$$

However, our main interest is to obtain global bounds in $x$, which we shall phrase through the following problem.

Problem 1. A 2-weight problem for the operator $P_{t_{0}}^{*}$. Given $1<p<\infty$, characterize the class of weights $w(x)>0$ such that $P_{t_{0}}^{*}$ maps $L^{p}(w) \rightarrow L^{p}(v)$ boundedly, for some other weight $v(x)>0$.

Our second main result gives a complete answer to Problem 1. For $p \in(1, \infty)$ we define the class of weights

$$
D_{p}(\Phi)=\left\{w(y)>0:\left\|w^{-\frac{1}{p}} \Phi\right\|_{L^{p^{\prime}\left(\mathbb{R}_{+}\right)}}<\infty\right\} .
$$

Observe that $L^{p}(w) \subset L^{1}(\Phi)$ if and only if $w \in D_{p}(\Phi)$, so in view of Theorem 1.1, this is a necessary condition for Problem 1 . Our second theorem shows that it is also sufficient.

Theorem 1.2. Let $1<p<\infty$ and $t_{0}>0$ be fixed. Then, for a weight $w(x)>0$ the condition $w \in D_{p}(\Phi)$ is equivalent to the existence of some other weight $v(x)>0$ such that

$$
P_{t_{0}}^{*}: L^{p}(w) \rightarrow L^{p}(v) \quad \text { boundedly. }
$$

Moreover, for every $\varepsilon>0$, we can choose a weight $v \in D_{p+\varepsilon}(\Phi)$ satisfying (1.7).

We remark that Problem 1 is only a "one-side" problem, in contrast with the (more difficult) question of characterizing all pairs of weights $(w, v)$ for which (1.7) holds. One-side problems were considered in the early 80s by Rubio de Francia [11] and Carleson and Jones [1] for various classical operators. Here we shall follow the approach by the latter, which has the advantage of giving explicit expressions for the second weight $v(x)$ (see Remark 6.2 below). This is also a novelty compared to [5], where we used the non-constructive method of Rubio de Francia.

We can now briefly describe our approach to the proofs in this paper. Rather than working with the operator $\mathbb{L}$, most of our computations will involve the "squared" Laguerre operator

$$
L=-\partial_{y y}+\left[y^{2}+\frac{\alpha^{2}-\frac{1}{4}}{y^{2}}\right]+2 \mu .
$$

This has the advantage of resembling the Hermite operator (which is the case $\alpha=-1 / 2$ ), so at some points we may use computations from [5]. There are however various additional difficulties which are characteristic of the Laguerre setting. The term $1 / y^{2}$ produces a singularity when $y \rightarrow 0$ which must be handled separately from the singularity at $y \rightarrow$ $\infty$. This is reflected in the behavior of the Bessel function $I_{\alpha}$ which is part of the kernel expression of $e^{-u L}$. One may also expect additional difficulties when $\alpha \in(-1,-1 / 2)$ (cases 
sometimes avoided in the literature, but that we consider here), related to the fact that such Laguerre functions blow-up when $y \rightarrow 0$.

Most of our work will be employed in deriving precise decay estimates for the Poisson kernel, which will lead to the following control of the operator $P_{t_{0}}^{*}$

$$
P_{t_{0}}^{*} f(x) \lesssim C(x)\left[\mathcal{M}^{\mathrm{loc}}(f \Phi)(x)+\int_{\mathbb{R}_{+}} f \Phi\right]
$$

for a reasonably well-behaved $C(x)$ (to be absorbed later as part of the weight $v(x)$ ). Here $\mathcal{M}^{\text {loc }}$ denotes a local Hardy-Littlewood maximal operator in $\mathbb{R}_{+}$, given by

$$
\mathcal{M}^{\mathrm{loc}} f(x):=\sup _{r>0} \frac{1}{|I(x, r)|} \int_{I(x, r)}|f(y)| \chi_{\left\{\frac{x}{2} \leq y \leq M x\right\}} d y
$$

for a suitable $M>1$. We also use the notation $I(x, r)=(x-r, x+r) \cap \mathbb{R}_{+}$.

Finally, we remark that the statement of Theorems 1.1 and 1.2 remains true when $\mathbb{L}$ is replaced by any of the Laguerre-type operators in the table below, provided $\Phi$ in (1.4) is replaced by the corresponding function in the table. As in (1.4), in the extremal case

\begin{tabular}{|c|c|c|}
\hline Eigenfunctions & differential operator & function $\Phi$ \\
\hline$L_{n}^{\alpha}$ & $\left.\begin{array}{r}\mathbb{L}=-y \partial_{y y}-(\alpha+1-y) \partial_{y}+m \\
m \geq 0\end{array}\right\}$ & $\frac{y^{\alpha} e^{-y}}{(1+y)^{m}[\ln (e+y)]^{1+\nu}}$ \\
\hline$\varphi_{n}^{\alpha}$ & $\left.\begin{array}{r}L=-\partial_{y y}+y^{2}+\frac{1}{y^{2}}\left(\alpha^{2}-\frac{1}{4}\right)+2 \mu \\
\mu \geq-(\alpha+1)\end{array}\right\}$ & $\frac{y^{\alpha+\frac{1}{2}} e^{-y^{2} / 2}}{(1+y)^{1+\alpha+\mu}[\ln (e+y)]^{1+\nu}}$ \\
\hline $\mathfrak{L}_{n}^{\alpha}$ & $\left.\begin{array}{r}\mathfrak{L}=-y \partial_{y y}-\partial_{y}+\frac{1}{4}\left[y+\frac{\alpha^{2}}{y}\right]+\frac{\mu}{2} \\
\mu \geq-(\alpha+1)\end{array}\right\}$ & $\frac{y^{\frac{\alpha}{2}} e^{-y / 2}}{(1+y)^{\frac{1+\alpha+\mu}{2}}[\ln (e+y)]^{1+\nu}}$ \\
\hline$\ell_{n}^{\alpha}$ & $\left.\begin{array}{r}\mathscr{L}=-y \partial_{y y}-(\alpha+1) \partial_{y}+\frac{y}{4}+\frac{\mu}{2} \\
\mu \geq-(\alpha+1)\end{array}\right\}$ & $\frac{y^{\alpha} e^{-y / 2}}{(1+y)^{\frac{1+\alpha+\mu}{2}}[\ln (e+y)]^{1+\nu}}$ \\
\hline$\psi_{n}^{\alpha}$ & $\left.\begin{array}{r}\Lambda=-\partial_{y y}-\frac{2 \alpha+1}{y} \partial_{y}+y^{2}+2 \mu \\
\mu \geq-(\alpha+1)\end{array}\right\}$ & $\frac{y^{2 \alpha+1} e^{-y^{2} / 2}}{(1+y)^{1+\alpha+\mu}[\ln (e+y)]^{1+\nu}}$ \\
\hline
\end{tabular}
$\mu=-(\alpha+1)$, the logarithmic term in the denominator of $\Phi$ must be replaced by $[\log (e+y)]^{\nu}$.

TABLE 1. Table of $\Phi$-functions for various Laguerre-type operators. 
The outline of the paper will be the following. In 92 we consider a version of Theorem 1.1 for heat integrals $u(t, x)=e^{-t L} f(x)$, which are solutions of the heat equation

$$
u_{t}+L u=0 \quad \text { in }(0, \mathrm{~T}) \times \mathbb{R}_{+}, \quad \text { with } \quad u(0, x)=f(x) .
$$

Heat integrals are easier to handle, and the explicit expression of the heat kernel, $e^{-t L}(x, y)$, makes more transparent the behavior we shall later encounter in Poisson kernels. In $₫ 3$ we study 2 -weight inequalities for the local maximal operator $\mathcal{M}^{\text {loc }}$. In $\$ 4$ we apply these to prove a version of Theorem 1.2 for heat integrals. In $\$ 5$ we take up the study of Poisson integrals, splitting in various subsections the detailed kernel estimates leading to (1.9). In \$6 we shall give the proof of Theorems 1.1 and 1.2 for the operator $L$. Finally, in 97 we show how to transfer the results to the Laguerre operators in Table 1.

Throughout the paper $\alpha>-1$ is fixed, as are the parameters $\mu, m$ in the differential operators. The notation $A \lesssim B$ will mean $A \leq c B$, for a constant $c>0$ which may depend on $\alpha, \mu$ and other parameters like $p, M, t_{0}, \varepsilon$, but not on $t, x, y$. If needed, we shall stress the latter dependence by $c(x), c(t, x), \ldots$ Finally, if $1<p<\infty$ we set $p^{\prime}=p /(p-1)$.

\section{The Simpler MODEL OF HeAT INTEGRALS}

In this section $L$ will denote the Laguerre-type operator

$$
L=-\partial_{y y}+\left[y^{2}+\frac{\alpha^{2}-\frac{1}{4}}{y^{2}}\right]
$$

that is, we have set $\mu=0$ in (1.8)*. The corresponding eigenfunctions $\left\{\varphi_{n}^{\alpha}\right\}_{n=0}^{\infty}$ satisfy

$$
L \varphi_{n}^{\alpha}=(4 n+2 \alpha+2) \varphi_{n}^{\alpha}, \quad n=0,1,2, \ldots
$$

and form an orthonormal basis of $L^{2}(0, \infty)$. The kernel of the associated heat semigroup $e^{-t L}$, written in terms of the new variable $s=$ th $t$, has the explicit expression

$$
\begin{aligned}
e^{-t L}(x, y) & =\sum_{n=0}^{\infty} e^{-(4 n+2 \alpha+2) t} \varphi_{n}^{\alpha}(x) \varphi_{n}^{\alpha}(y) \\
& =\sqrt{\frac{1-s^{2}}{2 s}} \bar{I}_{\alpha}\left(\frac{\left(1-s^{2}\right) x y}{2 s}\right) e^{-\frac{(x-y)^{2}}{4 s}} e^{-\frac{s(x+y)^{2}}{4}}
\end{aligned}
$$

Here we have used the convenient notation $\bar{I}_{\alpha}(z)=\sqrt{z} e^{-z} I_{\alpha}(z)$, so that $\bar{I}_{\alpha}(z) \approx\langle z\rangle^{\alpha+\frac{1}{2}}$, with $l z=\min \{z, 1\}$.

2.1. A.e. convergence of heat integrals. We wish to establish the pointwise convergence of $e^{-t L} f(x)$ with the weakest possible conditions in $f$. For this purpose, the following kernel bound will suffice

$$
e^{-t L}(x, y) \lesssim\left\langle\frac{x y}{s}\right\rangle^{\alpha+\frac{1}{2}} \frac{e^{-\frac{(x-y)^{2}}{4 s}}}{\sqrt{s}}
$$

${ }^{*}$ For heat integrals this implies no loss, since the general case can be factored as $e^{-2 \mu t}\left[e^{-t L} f\right](x)$. 
To produce this bound from (2.2) one disregards the last exponential, and uses $1-s^{2} \leq 1$ when $\alpha \geq-\frac{1}{2}$. If $\alpha \in\left(-1,-\frac{1}{2}\right)$, note that $l \lambda z \geq \lambda l z$ for $\lambda \leq 1$, so one can leave outside a power $\left(1-s^{2}\right)^{\alpha+1} \leq 1$.

Theorem 2.1. Let $\alpha>-1$ be fixed, and $f$ be such that

$$
\int_{0}^{\infty}|f(y)| e^{-a y^{2}} l y^{\alpha+\frac{1}{2}} d y<\infty, \quad \text { for some (possibly large) } a>0 .
$$

Then,

$$
\lim _{t \rightarrow 0^{+}} e^{-t L} f(x)=f(x), \quad \text { a.e. } x \in \mathbb{R}_{+} .
$$

Proof: For each fixed $N \geq 2$ it suffices to show that $\lim _{t \rightarrow 0^{+}} e^{-t L} f(x)=f(x)$ for a.e. $x \in(1 / N, N)$. We split

$$
f=f \chi_{\{0<y \leq 2 N\}}+f \chi_{\{y>2 N\}}=f_{1}+f_{2} .
$$

The function $f_{1}$ has bounded support and belongs to $L^{1}\left(y^{\alpha+\frac{1}{2}} e^{-\frac{y^{2}}{2}} d y\right)$, so we can apply the results of Muckenhoupt [8] (with a suitable change of variablest, as indicated by Stempak [12]) to obtain

$$
\lim _{t \rightarrow 0^{+}} e^{-t L} f_{1}(x)=f_{1}(x)=f(x), \quad \text { a.e. } x \in\left[\frac{1}{N}, N\right] .
$$

Next we shall show that, under the hypothesis (2.4),

$$
\lim _{t \rightarrow 0^{+}} e^{-t L} f_{2}(x)=0, \quad \forall x \in\left[\frac{1}{N}, N\right] .
$$

Since $t \rightarrow 0$, we may assume that $s=$ th $t \leq s_{0}$ for some $s_{0}<\frac{1}{10}$ (which we shall make precise below). Note that $\frac{1}{N} \leq x \leq N$ and $y>2 N$ imply that

$$
\left\langle\frac{x y}{s}\right\rangle^{\alpha+\frac{1}{2}}=1, \quad \forall s<1 .
$$

So, by (2.3), in this region we have a gaussian bound for the kernel

$$
e^{-t L}(x, y) \lesssim s^{-\frac{1}{2}} e^{-\frac{(x-y)^{2}}{4 s}} \leq s^{-\frac{1}{2}} e^{-\frac{y^{2}}{16 s}},
$$

using in the last step that $|x-y| \geq y / 2$. Choosing $s_{0}<\frac{1}{32 a}$ (with $a$ as in (2.4)), we see that for all $y>2 N$,

$$
e^{-t L}(x, y) \lesssim s^{-\frac{1}{2}} e^{-\frac{y^{2}}{32 s}} e^{-a y^{2}} \leq s^{-\frac{1}{2}} e^{-\frac{N^{2}}{8 s}} e^{-a y^{2}}
$$

and therefore

$$
e^{-t L} f_{2}(x) \lesssim s^{-\frac{1}{2}} e^{-\frac{N^{2}}{8 s}} \int_{y>2 N}|f(y)| e^{-a y^{2}} d y \longrightarrow 0, \quad \text { as } s \rightarrow 0^{+} .
$$

\footnotetext{
${ }^{\dagger}$ See e.g. $\oint \$ 7.2$ and 7.4 below for the explicit change of variables.
} 
2.2. Heat kernel estimates. The estimates in the proof of Theorem 2.1, slightly refined in some steps, lead to the following proposition.

Proposition 2.2. Let $\alpha>-1$. Then, for every $\gamma>1$ there is some $M=M_{\gamma}>1$ such that

$$
e^{-t L}(x, y) \leq C_{\gamma}\left\{\begin{array}{l}
\frac{e^{-\frac{|x-y|^{2}}{4 s}}}{\sqrt{s}}\left\langle\frac{x y}{s}\right\rangle^{\alpha+\frac{1}{2}} \quad \text { if } \quad \frac{x}{2} \leq y \leq M x \\
c(x)\langle y\rangle^{\alpha+\frac{1}{2}} e^{-\frac{y^{2}}{2 \gamma \operatorname{th}(2 t)}} \quad \text { if } y<\frac{x}{2} \quad \text { or } \quad y>M x
\end{array},\right.
$$

for all $x, y \in \mathbb{R}_{+}$and $s=\operatorname{th} t \in(0,1)$. Here we can $\operatorname{set} c(x)=1 / l x^{\alpha+\frac{3}{2}}$.

Proof: Given $x, y$ and $s$, for simplicity we write $z=\frac{x y}{s}$. Our estimates below will follow from

$$
e^{-t L}(x, y) \lesssim\langle z\rangle^{\alpha+\frac{1}{2}} s^{-\frac{1}{2}} e^{-\frac{(x-y)^{2}}{4 s}} e^{-\frac{s y^{2}}{4}}
$$

This clearly implies the estimate in the local part $y \in\left[\frac{x}{2}, M x\right]$, so we shall look at the complementary range. Below we shall ignore the last exponential factor in (2.6), and observe that all our estimates will end up with $e^{-y^{2} /(4 \gamma s)}$. Combining these two one obtains the asserted exponential bound, since

$$
e^{-\frac{y^{2}}{4 \gamma s}} e^{-\frac{s y^{2}}{4}} \leq e^{-\frac{y^{2}}{4 \gamma}\left(s+\frac{1}{s}\right)}=e^{-\frac{y^{2}}{2 \gamma \operatorname{th}(2 t)}}
$$

as $s+s^{-1}=2 / \operatorname{th}(2 t)$ when $s=\operatorname{th} t$.

To handle the kernel expression in (2.6) we need to separate the cases $z \leq 1$ and $z \geq 1$. We begin with $z \geq 1$. In the region $y>M x$ we may use $|x-y| \geq\left(1-\frac{1}{M}\right) y$ to obtain

$$
e^{-t L}(x, y) \lesssim \frac{e^{-\left(\frac{M-1}{M}\right)^{2} \frac{y^{2}}{4 s}}}{\sqrt{s}} \leq c_{M} \frac{e^{-\left(\frac{M-1}{M}\right)^{3} \frac{y^{2}}{4 s}}}{y} \leq c_{M} e^{-\frac{y^{2}}{4 s \gamma}} \frac{\langle y\rangle^{\alpha+\frac{1}{2}}}{l x^{\alpha+\frac{3}{2}}},
$$

where in the last step we select $M=M_{\gamma}$ sufficiently large so that $\left(\frac{M}{M-1}\right)^{3} \leq \gamma$, and have used the trivial estimate

$$
\frac{1}{y} \leq \frac{\langle y\rangle^{\alpha+\frac{1}{2}}}{l y^{\alpha+\frac{3}{2}}} \leq \frac{\langle y\rangle^{\alpha+\frac{1}{2}}}{l x^{\alpha+\frac{3}{2}}}, \quad \text { if } y \geq x
$$

On the other hand, if $y<x / 2$ we have $|x-y| \geq x / 2$, which leads to

$$
e^{-t L}(x, y) \lesssim \frac{e^{-\frac{(x / 2)^{2}}{4 s}}}{\sqrt{s}} \leq c_{\gamma} \frac{e^{-\frac{(x / 2)^{2}}{4 s \gamma}}}{x} \leq c_{\gamma} \frac{e^{-\frac{y^{2}}{4 \gamma s}}}{x} .
$$

In the case $\alpha \in\left(-1,-\frac{1}{2}\right)$ this can be combined with

$$
\frac{1}{x} \leq \frac{\langle x\rangle^{\alpha+\frac{1}{2}}}{l x^{\alpha+\frac{3}{2}}} \leq \frac{\langle y\rangle^{\alpha+\frac{1}{2}}}{l x^{\alpha+\frac{3}{2}}}, \quad \text { since } x \geq y .
$$


If on the contrary $\alpha \geq-\frac{1}{2}$, we can insert $1 \leq z^{\alpha+\frac{1}{2}}$ in the gaussian bound and obtain

$$
e^{-t L}(x, y) \lesssim \frac{e^{-\frac{(x / 2)^{2}}{4 s}}}{\sqrt{s}}\left(\frac{x y}{s}\right)^{\alpha+\frac{1}{2}}=\left(\frac{x^{2}}{s}\right)^{\alpha+1} e^{-\frac{(x / 2)^{2}}{4 s}} \frac{y^{\alpha+\frac{1}{2}}}{x^{\alpha+\frac{3}{2}}} \leq c_{\gamma} e^{-\frac{y^{2}}{4 s \gamma}} \frac{\langle y\rangle^{\alpha+\frac{1}{2}}}{l x^{\alpha+\frac{3}{2}}} .
$$

This completes the proof of (2.5) when $z \geq 1$.

We turn to the case $z \leq 1$, and replace the gaussian bound by

$$
e^{-t L}(x, y) \lesssim s^{-\frac{1}{2}} e^{-\frac{(x-y)^{2}}{4 s}} z^{\alpha+\frac{1}{2}} .
$$

This is a better bound when $\alpha \geq-\frac{1}{2}$, so some of the previous arguments also lead to (2.5); namely one can disregard $z$ in the region $y>M x$, and must keep it when $y<\frac{x}{2}$ and argue as in (2.9). We are left with the case $\alpha \in\left(-1,-\frac{1}{2}\right)$, which makes $z^{\alpha+\frac{1}{2}} \geq 1$. In the region $y>M x$, this can be absorbed by the exponentials since

$$
\begin{aligned}
e^{-t L}(x, y) & \lesssim\left(\frac{x y}{s}\right)^{\alpha+\frac{1}{2}} s^{-\frac{1}{2}} e^{-\left(\frac{M-1}{M}\right)^{2} \frac{y^{2}}{4 s}}=\left(\frac{y^{2}}{s}\right)^{\alpha+1} e^{-\left(\frac{M-1}{M}\right)^{2} \frac{y^{2}}{4 s}} \frac{x^{\alpha+\frac{1}{2}}}{y^{\alpha+\frac{3}{2}}} \\
& \leq c_{M} e^{-\left(\frac{M-1}{M}\right)^{3} \frac{y^{2}}{4 s}} \frac{\langle x\rangle^{\alpha+\frac{1}{2}}\langle y\rangle^{\alpha+\frac{1}{2}}}{l y^{2 \alpha+2}} \leq c_{M} e^{-\frac{y^{2}}{4 s \gamma}} \frac{\langle x\rangle^{\alpha+\frac{1}{2}}\langle y\rangle^{\alpha+\frac{1}{2}}}{l x^{2 \alpha+2}},
\end{aligned}
$$

using in the last step that $y \geq x$. Finally, in the region $y<\frac{x}{2}$ the inequalities in (2.9) remain also valid, so we have completed the proof of Proposition 2.2.

Proposition 2.2 can be expressed in terms of the local Hardy-Littlewood maximal function in $\mathbb{R}_{+}$

$$
\mathcal{M}_{M}^{\mathrm{loc}} f(x):=\sup _{r>0} \frac{1}{|I(x, r)|} \int_{I_{r}(x)}|f(y)| \chi_{\left\{\frac{x}{2}<y<M x\right\}} d y,
$$

where $I(x, r)=I_{r}(x)$ denotes the interval $(x-r, x+r) \cap \mathbb{R}_{+}$.

Corollary 2.3. Let $\alpha>-1$ and $\gamma>1$. Then there is some $M=M_{\gamma}>1$ such that

$$
\sup _{0<t \leq t_{0}}\left|e^{-t L} f(x)\right| \lesssim \mathcal{M}_{M}^{\text {loc }} f(x)+c(x) \int_{\mathbb{R}_{+}}|f(y)|\langle y\rangle^{\alpha+\frac{1}{2}} e^{-\frac{y^{2}}{2 \gamma t \mathrm{th}\left(2 t_{0}\right)}} d y,
$$

for every $x, y, t_{0}>0$ and $c(x)=1 / l x^{\alpha+\frac{3}{2}}$.

Proof: We only have to prove the local estimate, and may assume that supp $f \subset\left[\frac{x}{2}, M x\right]$. If $s=\operatorname{th} t \leq x^{2}$, then $z=\frac{x y}{s} \gtrsim 1$ (since $x \approx y$ when $y \in \operatorname{supp} f$ ), so the gaussian bound of the kernel and a standard slicing argument easily lead to

$$
\left|e^{-t L} f(x)\right| \lesssim \frac{1}{\sqrt{s}} \int_{\mathbb{R}_{+}} e^{-\frac{(x-y)^{2}}{4 s}}|f(y)| \chi_{\left\{\frac{x}{2} \leq y \leq M x\right\}} d y \lesssim \mathcal{M}_{M}^{\text {loc }} f(x) .
$$

If $s=$ th $t \geq x^{2}$, then using $x \approx y$,

$$
\left|e^{-t L} f(x)\right| \lesssim \frac{x^{2 \alpha+1}}{s^{\alpha+1}} \int_{\frac{x}{2}}^{M x}|f(y)| d y \lesssim \frac{x^{2 \alpha+1}}{x^{2 \alpha+2}} \int_{\frac{x}{2}}^{M x}|f(y)| d y \lesssim \mathcal{M}_{M}^{\text {loc }} f(x) .
$$

Remark 2.4. An estimate quite similar to (2.12), with a slightly worse bound for the exponential inside the integral, was obtained by Chicco-Ruiz and Harboure in [2, §5]. 


\section{2-Weight INEQUALities FOR $\mathcal{M}^{\text {loc }}$}

This section is about the local maximal operator in $\mathbb{R}_{+}$

$$
\mathcal{M}^{\text {loc }} f(x):=\sup _{t>0} \frac{1}{\left|I_{t}(x)\right|} \int_{I_{t}(x)}|f(y)| \chi_{\left\{\frac{x}{2}<y<M x\right\}} d y,
$$

where $M>1$ is a fixed parameter. For simplicity, we do not include the subscript $M$ in the notation, but the implicit constants appearing below will all depend on $M$. For the 1-weight theory of this operator we refer to [10, §6].

For each $p \in(1, \infty)$, consider the following family of weights in $\mathbb{R}_{+}$

$$
D_{p}^{\mathrm{loc}}=\left\{W(x)>0: \int_{J} W^{-\frac{p^{\prime}}{p}}<\infty, \quad \forall J \Subset(0, \infty)\right\} .
$$

Associated with $W \in D_{p}^{\text {loc }}$, we consider a family of weights $\left\{V_{\varepsilon}\right\}_{\varepsilon>0}$, defined by

$$
V_{\varepsilon}(x)=V(x) \rho_{\varepsilon}[V(x)], \quad \text { where } V(x):=\left[\mathcal{M}^{\operatorname{loc}}\left(W^{-\frac{p^{\prime}}{p}}\right)(x)\right]^{-\frac{p}{p^{\prime}}},
$$

and with the notation $\rho_{\varepsilon}(x):=\min \left\{x^{\varepsilon}, x^{-\varepsilon}\right\}$. Observe that $V_{\varepsilon_{2}} \leq V_{\varepsilon_{1}} \leq V \leq W$ if $\varepsilon_{1} \leq \varepsilon_{2}$. This definition is a slight variant of the one proposed by Carleson and Jones in [1], and leads to the following 2 -weight inequalities.

Theorem 3.1. Let $1<p<\infty$ and $W \in D_{p}^{\text {loc }}$. Then for every $\varepsilon>0$

$$
\mathcal{M}^{\mathrm{loc}}: L^{p}(W) \rightarrow L^{p}\left(V_{\varepsilon}\right) \text { boundedly, }
$$

where $V_{\varepsilon}$ is defined as in (3.1).

Proof: The argument of the proof is due to Carleson and Jones [1] (see also a recent application in [4, Prop. 4.2]). For completeness, we sketch the modifications required for the local operator $\mathcal{M}^{\text {loc }}$. Call $E_{n}=\left\{x \in \mathbb{R}_{+}: \mathcal{M}^{\text {loc }}\left(W^{-\frac{1}{p-1}}\right)(x)<2^{n}\right\}, n \in \mathbb{Z}$, and define the operators

$$
T_{n} g(x):=\chi_{E_{n}} \mathcal{M}^{\mathrm{loc}}\left(W^{-\frac{1}{p-1}} g\right)(x)
$$

Note that $T_{n}: L^{1}\left(W^{-\frac{1}{p-1}}\right) \rightarrow L^{1, \infty}$, with a uniform bound in $n$, since

$$
\left|\left\{T_{n} g(x)>R\right\}\right| \leq\left|\left\{\mathcal{M}\left(W^{-\frac{1}{p-1}} g\right)(x)>R\right\}\right| \leq \frac{c_{0}}{R} \int_{\mathbb{R}_{+}} W^{-\frac{1}{p-1}}|g|,
$$

using in the last step the weak-1 boundedness of the Hardy-Littlewood maximal operator. Similarly, $T_{n}: L^{\infty}\left(W^{-\frac{1}{p-1}}\right) \rightarrow L^{\infty}$ with $\left\|T_{n}\right\| \leq 2^{n}$, since

$$
\left\|T_{n} g\right\|_{\infty}=\sup _{x \in E_{n}}\left|\mathcal{M}^{\mathrm{loc}}\left(W^{-\frac{1}{p-1}} g\right)(x)\right| \leq 2^{n}\|g\|_{\infty} \text {. }
$$

Thus, by the Marcinkiewicz interpolation theorem we obtain

$$
\int_{E_{n}}\left|T_{n}(g)\right|^{p} \leq c_{0} 2^{\frac{n p}{p^{\prime}}} \int_{\mathbb{R}^{d}}|g|^{p} W^{-\frac{1}{p-1}}, \quad n \in \mathbb{Z} .
$$

Setting $g=f W^{\frac{1}{p-1}}$ in the above inequality, this is the same as

$$
\int_{E_{n}}\left|\mathcal{M}^{\mathrm{loc}}(f)\right|^{p} \leq c_{0} 2^{\frac{n p}{p^{\prime}}} \int_{\mathbb{R}_{+}}|f|^{p} W, \quad n \in \mathbb{Z} .
$$


Now, modulo null sets $\mathbb{R}_{+}=\cup_{n \in \mathbb{Z}}\left[E_{n} \backslash E_{n-1}\right]$ (since $0<\mathcal{M}^{\text {loc }}\left(W^{-\frac{1}{p-1}}\right)(x)<\infty$ at a.e. $x$ ), and we have

$$
V_{\varepsilon}(x) \approx 2^{-\frac{n p}{p^{\prime}}} 2^{-\frac{\varepsilon|n| p}{p^{\prime}}}, \text { if } x \in E_{n} \backslash E_{n-1} .
$$

Therefore, we obtain

$$
\begin{aligned}
\int_{\mathbb{R}_{+}}\left|\mathcal{M}^{\text {loc }} f\right|^{p} V_{\varepsilon} & \lesssim \sum_{n \in \mathbb{Z}} 2^{-\frac{n p}{p^{\prime}}} 2^{-\frac{\varepsilon|n| p}{p^{\prime}}} \int_{E_{n}}\left|\mathcal{M}^{\text {loc }} f\right|^{p} \\
\text { (by (3.6) }) & \lesssim\left(\sum_{n \in \mathbb{Z}} 2^{-\frac{\varepsilon|n| p}{p^{\prime}}}\right) \int_{\mathbb{R}^{d}}|f|^{p} W
\end{aligned}
$$

as we wished to show.

The weight $V_{\varepsilon}$ inherits some of the integrability behavior of $W$ if $\varepsilon$ is sufficiently small. To state this we first define the subclasses

$$
\begin{aligned}
D_{p}^{0}(\beta) & =\left\{W \in D_{p}^{\mathrm{loc}}: \int_{0}^{1} W^{-\frac{p^{\prime}}{p}}(y) l y^{\beta p^{\prime}} d y<\infty\right\}, \quad \text { for } \beta>-1, \\
D_{p}^{\exp }(a) & =\left\{W \in D_{p}^{\mathrm{loc}}: \int_{1}^{\infty} W^{-\frac{p^{\prime}}{p}}(y) e^{-a y^{2} p^{\prime}} d y<\infty\right\}, \quad \text { for } a>0 .
\end{aligned}
$$

Proposition 3.2. Let $1<p<\infty$ and $W \in D_{p}^{\text {loc }}$. Then, for each $\varepsilon>0$, the weight defined in (3.1) satisfies $V_{\varepsilon} \in D_{q}^{\text {loc }}$, for all $q>p+\varepsilon p / p^{\prime}$. Moreover, we additionally have

(i) $W \in D_{p}^{0}(\beta)$ implies $V_{\varepsilon} \in D_{q}^{0}(\beta)$, provided $q>p+\varepsilon \frac{p}{p^{\prime}} \frac{\left|1+\beta p^{\prime}\right|}{1+\beta}$

(ii) $W \in D_{p}^{\exp }(a)$ implies $V_{\varepsilon} \in D_{q}^{\exp }(b)$, provided $q>p(1+\varepsilon) M^{2} a / b$.

PRoof: Observe that

$$
V_{\varepsilon}(x)^{-\frac{q^{\prime}}{q}}=\max _{ \pm}\left[\mathcal{M}^{\mathrm{loc}}\left(W^{-\frac{1}{p-1}}\right)(x)\right]^{\frac{p-1}{q-1}(1 \pm \varepsilon)}
$$

The assumption $q>p+\frac{\varepsilon p}{p^{\prime}}$ implies that $s=\frac{(p-1)(1+\varepsilon)}{q-1}<1$. Then, given $J=[a, b] \Subset \mathbb{R}_{+}$,

$$
\begin{aligned}
\int_{J}\left[\mathcal{M}^{\mathrm{loc}}\left(W^{-\frac{1}{p-1}}\right)\right]^{s} & \lesssim|J|^{1-s}\left\|\mathcal{M}\left(W^{-\frac{1}{p-1}} \chi_{J^{*}}\right)\right\|_{L^{1, \infty}}^{s} \\
& \lesssim c_{J}\left(\int_{J^{*}} W^{-\frac{1}{p-1}}\right)^{s}<\infty,
\end{aligned}
$$

with $J^{*}=[a / 2, M b] \Subset(0, \infty)$. The same applies if we set $s=\frac{(p-1)(1-\varepsilon)}{q-1}($ which is also $<1)$, so we deduce from (3.7) that $\int_{J} V_{\varepsilon}^{-\frac{1}{q-1}}<\infty$. 
We next prove (i), and as before set $s=\frac{(p-1)(1+\varepsilon)}{q-1}<1$. Then, denoting $I_{j}=\left[2^{-j-1}, 2^{-j}\right]$, we have

$$
\begin{aligned}
& \int_{0}^{1}\left[\mathcal{M}^{\mathrm{loc}}\left(W^{-\frac{1}{p-1}}\right)\right]^{s} l y^{\beta q^{\prime}} d y \lesssim \sum_{j=0}^{\infty} 2^{-j \beta q^{\prime}}\left|I_{j}\right|^{1-s}\left\|\mathcal{M}\left(W^{-\frac{1}{p-1}} \chi_{I_{j}^{*}}\right)\right\|_{L^{1, \infty}}^{s} \\
& \lesssim \sum_{j=0}^{\infty} 2^{-j \beta q^{\prime}} 2^{-j(1-s)}\left(\int_{2^{-j-2}}^{M 2^{-j}} W^{-\frac{1}{p-1}}\right)^{s} \\
& \lesssim \sum_{j=0}^{\infty} 2^{-j\left[\beta\left(q^{\prime}-p^{\prime} s\right)+1-s\right]}\left(\int_{0}^{M} W^{-\frac{1}{p-1}}(y) l y^{\beta p^{\prime}} d y\right)^{s} .
\end{aligned}
$$

This is a finite expression provided

$$
\beta\left(q^{\prime}-p^{\prime} s\right)+1-s>0,
$$

and using the value of $s=\frac{(p-1)(1+\varepsilon)}{q-1}$ and solving for $q$ this is equivalent to

$$
q>p+\frac{\varepsilon p\left(1+\beta p^{\prime}\right)}{p^{\prime}(1+\beta)} .
$$

In order to have $\int_{0}^{1} V_{\varepsilon}^{-\frac{1}{q-1}} l y^{\beta q^{\prime}} d y<\infty$ the previous relation must also hold with $\varepsilon$ replaced by $-\varepsilon$, so a sufficient condition is

$$
q>p+\frac{\varepsilon p\left|1+\beta p^{\prime}\right|}{p^{\prime}(1+\beta)}
$$

as we wished to show.

We now prove (ii). Let $\gamma>1$ (to be precised later), and as before set $I_{j}=\left[\gamma^{j}, \gamma^{j+1}\right]$ and $s=\frac{(p-1)(1+\varepsilon)}{q-1}<1$. Then

$$
\begin{aligned}
\int_{1}^{\infty}\left[\mathcal{M}^{\mathrm{loc}}\left(W^{-\frac{1}{p-1}}\right)\right]^{s} & e^{-b y^{2} q^{\prime}} d y \lesssim \sum_{j=0}^{\infty} e^{-b \gamma^{2 j} q^{\prime}} \gamma^{(1-s) j}\left\|\mathcal{M}\left(W^{-\frac{1}{p-1}} \chi_{I_{j}^{*}}\right)\right\|_{L^{1, \infty}}^{s} \\
& \lesssim \sum_{j=0}^{\infty} e^{-b \gamma^{2 j} q^{\prime}} \gamma^{(1-s) j}\left(\int_{\gamma^{j} / 2}^{M \gamma^{j+1}} W^{-\frac{1}{p-1}} d y\right)^{s} \\
& \leq \sum_{j=0}^{\infty} \gamma^{(1-s) j} e^{-\gamma^{2 j}\left[b q^{\prime}-p^{\prime} a M^{2} \gamma^{2} s\right]}\left(\int_{1 / 2}^{\infty} W^{-\frac{1}{p-1}} e^{-p^{\prime} a y^{2}} d y\right)^{s} .
\end{aligned}
$$

This is now a finite expression provided

$$
b q^{\prime}>p^{\prime} a M^{2} \gamma^{2} s,
$$

which using the value of $s$ and solving for $q$ gives

$$
q>p(1+\varepsilon) M^{2} \gamma^{2} a / b .
$$

Clearly, we can choose a $\gamma>1$ with this property under the assumption

$$
q>p(1+\varepsilon) M^{2} a / b .
$$

Since this also implies the validity of the estimates with $\varepsilon$ replaced by $-\varepsilon$, we may conclude that $V_{\varepsilon} \in D_{q}^{\exp }(b)$, as desired. 


\section{2-WEIGHT INEQUALITIES FOR LOCAL MAXIMAL HEAT OPERATORS}

Let $L$ be as in (2.1), and for each $t_{0}>0$, consider

$$
h_{t_{0}}^{*} f(x):=\sup _{0<t \leq t_{0}}\left|e^{-t L} f(x)\right| .
$$

Given any $\mathrm{T}>t_{0}$, this operator is well defined over functions $f \in L^{1}\left(\varphi_{\mathrm{T}}\right)$, where

$$
\varphi_{\mathrm{T}}(y)=\langle y\rangle^{\alpha+\frac{1}{2}} e^{-\frac{y^{2}}{2 \operatorname{th}(2 \mathrm{~T})}} .
$$

We wish to study 2-weight inequalities for $h_{t_{0}}^{*}$ over subspaces $L^{p}(w) \subset L^{1}\left(\varphi_{\mathrm{T}}\right)$. By duality, the class of weights for which such inclusion holds is given by

$$
D_{p}\left(\varphi_{\mathrm{T}}\right):=\left\{w>0:\left\|w^{-\frac{1}{p}} \varphi_{\mathrm{T}}\right\|_{p^{\prime}}<\infty\right\}
$$

Here we show that for all such weights the operator $h_{t_{0}}^{*}$ satisfies a 2 -weight inequality.

Theorem 4.1. Let $\mathrm{T}>t_{0}>0$ and $1<p<\infty$. Then, for every $w \in D_{p}\left(\varphi_{\mathrm{T}}\right)$ there exists another weight $v(x)>0$ such that

$$
h_{t_{0}}^{*}: L^{p}(w) \rightarrow L^{p}(v), \quad \text { boundedly }
$$

Moreover, if $q>p$ and $t_{0}$ is sufficiently small, then we can select $v \in D_{q}\left(\varphi_{\mathrm{T}}\right)$.

Remark 4.2. The second weight $v(x)$ will be constructed explicitly; see (4.2), (4.5) and (4.6) below. Observe that $v$ depends on $\alpha, p, t_{0}, \mathrm{~T}$ and of course $w$.

ProOF of Theorem 4.1; $\quad$ The crucial estimate was already given in Corollary 2.3, We shall use it with the parameter $\gamma=\operatorname{th}(2 \mathrm{~T}) / \operatorname{th}\left(2 t_{0}\right)>1$, which produces a suitable $M=M_{\gamma}>1$ such that

$$
h_{t_{0}}^{*} f(x) \lesssim \mathcal{M}_{M}^{\mathrm{loc}} f(x)+c(x) \int_{0}^{\infty}|f(y)| \varphi_{\mathrm{T}}(y) d y .
$$

The last integral is bounded by $\|f\|_{L^{p}(w)}\left\|w^{-\frac{1}{p}} \varphi_{\mathrm{T}}\right\|_{p^{\prime}}$, so the second term will be fine for any weight $v(x)$ such that $c(x)=1 /\langle x\rangle^{\alpha+\frac{3}{2}} \in L^{p}(v)$. For instance we may take

$$
v_{2}(x)=\frac{l x^{\left(\alpha+\frac{3}{2}\right) p-1}}{[\log (e / l x)]^{2}(1+x)}
$$

which clearly satisfies

$$
\int_{0}^{\infty}|c(x)|^{p} v_{2}(x) d x=\int_{0}^{\infty} \frac{d x}{l x[\log (e / l x)]^{2}(1+x)^{p}}<\infty .
$$

Further, we claim that $v_{2} \in D_{q}\left(\varphi_{\mathrm{T}}\right)$ iff $q>p$. Indeed, since $\varphi_{\mathrm{T}}(x)$ decays exponentially, it suffices to check the integrability for $x$ near 0 . Writing $\beta=\alpha+\frac{1}{2}$ so that

$$
\varphi_{\mathrm{T}}(x) \approx l x^{\beta} \quad \text { and } \quad v_{2}(x) \approx \frac{l x^{(\beta+1) p-1}}{[\log (e / l x)]^{2}}
$$


we easily see that

$$
\int_{0}^{1} v_{2}(x)^{-\frac{q^{\prime}}{q}} l x^{\beta q^{\prime}} d x \approx \int_{0}^{1} \frac{[\log (e / x)]^{2 q^{\prime} / q}}{x^{1-q^{\prime}(\beta+1)\left(1-\frac{p}{q}\right)}} d x<\infty .
$$

For the first term in (4.1) we shall use the results in $\$ 3$. We first note that

$$
w \in D_{p}\left(\varphi_{\mathrm{T}}\right) \Longleftrightarrow w \in D_{p}^{0}\left(\alpha+\frac{1}{2}\right) \cap D_{p}^{\exp }(a), \quad \text { with } a=1 /(2 \text { th } 2 \mathrm{~T})
$$

where the weight classes $D_{p}^{0}(\beta)$ and $D_{p}^{\text {exp }}(a)$ were defined just before Proposition 3.2 . Then, for every $\varepsilon>0$ Theorem 3.1 gives

$$
\left\|\mathcal{M}_{M}^{\mathrm{loc}} f\right\|_{L^{p}\left(v_{1, \varepsilon}\right)} \lesssim\|f\|_{L^{p}(w)}
$$

provided

$$
v_{1, \varepsilon}(x)=\mathscr{V}(x) \rho_{\varepsilon}(\mathscr{V}(x)), \quad \text { where } \mathscr{V}(x)=\left[\mathcal{M}_{M}^{\text {loc }}\left(w^{\frac{1}{1-p}}\right)(x)\right]^{1-p}
$$

(or $v_{1, \varepsilon}=V_{\varepsilon}$ in the notation of (3.1) ). Hence setting

$$
v(x)=\min \left\{v_{1, \varepsilon}(x), v_{2}(x)\right\}
$$

with $v_{1, \varepsilon}$ and $v_{2}$ defined as in (4.5) and (4.2), we have proved that $h_{t_{0}}^{*}: L^{p}(w) \rightarrow L^{p}(v)$.

It remains to verify the last statement in Theorem 4.1. We already know that, for every $q>p$, we have $v_{2} \in D_{q}\left(\varphi_{\mathrm{T}}\right)$. Concerning $v_{1, \varepsilon}$, from the equivalence in (4.4) it suffices to prove that $V_{\varepsilon} \in D_{q}^{0}\left(\alpha+\frac{1}{2}\right) \cap D_{q}^{\exp }(a)$ for a sufficiently small $\varepsilon$. The first assertion is immediate from (i) in Proposition 3.2. However, (ii) in the same proposition only gives $V_{\varepsilon} \in D_{\rho}^{\exp }(a)$ if $\rho>p(1+\varepsilon) M^{2}$, where $M=M_{\gamma}$ is the parameter obtained in Proposition 2.2 by the rule $\left(\frac{M}{M-1}\right)^{3}=\gamma=\operatorname{th}(2 \mathrm{~T}) /$ th $\left(2 t_{0}\right)$. If we allow both $\varepsilon$ and $t_{0}$ be sufficiently small (so that $M$ becomes close enough to 1 ), then we can set $\rho=q$, and hence conclude that $v_{1, \varepsilon} \in D_{q}\left(\varphi_{\mathrm{T}}\right)$ as desired.

\section{Poisson Kernel estimates}

In this section we fix $\alpha>-1$ and $\mu \geq-(\alpha+1)$, and consider the Laguerre-type operator

$$
L=-\partial_{y y}+\left[y^{2}+\frac{\alpha^{2}-\frac{1}{4}}{y^{2}}\right]+2 \mu,
$$

whose eigenfunctions $\left\{\varphi_{n}^{\alpha}\right\}_{n=0}^{\infty}$ form an orthonormal basis of $L^{2}(0, \infty)$, and satisfy

$$
L \varphi_{n}^{\alpha}=(4 n+2(\alpha+1+\mu)) \varphi_{n}^{\alpha}, \quad n=0,1,2, \ldots
$$

They can be expressed in terms of the (normalized) Laguerre polynomials $L_{n}^{\alpha}$ by

$$
\varphi_{n}^{\alpha}(y)=\sqrt{2} y^{\alpha+\frac{1}{2}} e^{-\frac{y^{2}}{2}} L_{n}^{\alpha}\left(y^{2}\right)
$$


although we shall not use this formula here. The kernel of the associated heat semigroup, $e^{-t L}$, can be written explicitly in various forms

$$
\begin{aligned}
e^{-t L}(x, y) & =\sum_{n=0}^{\infty} e^{-[4 n+2(\alpha+1+\mu)] t} \varphi_{n}^{\alpha}(x) \varphi_{n}^{\alpha}(y) \\
\left(r=e^{-2 t}\right) & =r^{\mu} \sqrt{\frac{2 r}{1-r^{2}}} \bar{I}_{\alpha}\left(\frac{2 r x y}{1-r^{2}}\right) e^{-\frac{(x-r y)^{2}}{1-r^{2}}} e^{\frac{x^{2}-y^{2}}{2}} \\
(s=\text { th } t) & =\left(\frac{1-s}{1+s}\right)^{\mu} \sqrt{\frac{1-s^{2}}{2 s}} \bar{I}_{\alpha}\left(\frac{\left(1-s^{2}\right) x y}{2 s}\right) e^{-\frac{(x-y)^{2}}{4 s}} e^{-\frac{s(x+y)^{2}}{4}}
\end{aligned}
$$

where as before we have set $\bar{I}_{\alpha}(z)=\sqrt{z} e^{-z} I_{\alpha}(z)$. Thus, using the notation $l z=\min \{z, 1\}$, we shall have $\bar{I}_{\alpha}(z) \approx\langle z\rangle^{\alpha+\frac{1}{2}}$. Both expressions of the heat kernel will be useful in our later computations. For instance, (5.3) is good when $r \approx 0$, as it isolates correctly the decaying factor $\langle y\rangle^{\alpha+\frac{1}{2}} e^{-y^{2} / 2}$. On the other hand, (5.4) will be useful when $s \approx 0$ (hence $r \approx 1$ ), since it makes transparent the gaussian behavior of the singularity $s^{-\frac{1}{2}} e^{-\frac{(x-y)^{2}}{4 s}}$.

Using the subordination formula in (1.3), the Poisson kernel associated with $L$ becomes

$$
P_{t}(x, y):=\frac{t^{2 \nu}}{4^{\nu} \Gamma(\nu)} \int_{0}^{\infty} e^{-\frac{t^{2}}{4 u}}\left[e^{-u L}(x, y)\right] \frac{d u}{u^{1+\nu}}, \quad t>0 .
$$

Changing variables $r=e^{-2 u}$ (i.e., $u=\frac{1}{2} \ln \frac{1}{r}$ ) one sees that

$$
P_{t}(x, y) \approx t^{2 \nu} e^{\frac{x^{2}-y^{2}}{2}} \int_{0}^{1} e^{-\frac{t^{2}}{2 \ln \frac{1}{r}}} r^{\mu+\frac{1}{2}} \frac{e^{-\frac{(x-r y)^{2}}{1-r^{2}}}}{\sqrt{1-r}} \frac{\left\langle\frac{r x y}{1-r^{2}}\right\rangle^{\alpha+\frac{1}{2}}}{\left(\ln \frac{1}{r}\right)^{1+\nu}} \frac{d r}{r} .
$$

We shall consider two regions of integration according to the behavior of $z:=\frac{r x y}{1-r^{2}}$. The regions will be separated by the number

$$
r_{0}(x y)= \begin{cases}\frac{1}{2 x y} & , \text { if } x y \geq 1 \\ 1-\frac{x y}{2} & , \text { if } x y \leq 1 .\end{cases}
$$

Indeed, it is elementary to check that

(1) If $0<r \leq r_{0}(x y)$ then $z \leq 1$.

(2) If $r_{0}(x y) \leq r<1$ then $z \geq 1 / 2$.

Thus we can write

$$
\begin{aligned}
P_{t}(x, y) & \approx t^{2 \nu} e^{\frac{x^{2}-y^{2}}{2}}\left[\int_{0}^{r_{0}(x y)} \cdots\left(\frac{r x y}{1-r^{2}}\right)^{\alpha+\frac{1}{2}} \frac{d r}{r}+\int_{r_{0}(x y)}^{1} \cdots \frac{d r}{r}\right] \\
& =B_{t}(x, y)+A_{t}(x, y) .
\end{aligned}
$$

The next two propositions summarize the estimates we shall need to handle these kernels. We shall make extensive use of the function

$$
\Phi(y):=\frac{\langle y\rangle^{\alpha+\frac{1}{2}} e^{-y^{2} / 2}}{(1+y)^{\mu+\frac{1}{2}}[\log (y+e)]^{1+\nu}},
$$


with the agreement that in the extreme case $\mu=-(\alpha+1)$ the log in the denominator is just $[\log (y+e)]^{\nu}$. The first result gives, for fixed $t$ and $x$, the optimal decay of $y \mapsto P_{t}(x, y)$ in terms of the function $\Phi(y)$.

Proposition 5.1. Given $t, x>0$, there exist $c_{1}(t, x)>0$ and $c_{2}(t, x)>0$ such that

$$
c_{1}(t, x) \Phi(y) \leq P_{t}(x, y) \leq c_{2}(t, x) \Phi(y), \quad \forall y \in \mathbb{R}_{+} .
$$

The second result is a refinement of the upper bound in (5.7) with a few advantages: it is uniform in the variable $t$, it isolates in the "local part" the singularities of the kernel $P_{t}(x, y)$, and finally provides "reasonable" bounds for the constant's dependence on $x$.

Proposition 5.2. There exists $M>1$ such that the following holds for all $t, x, y>0$

$$
P_{t}(x, y) \lesssim C_{1}(x) \frac{t^{2 \nu} e^{-y^{2} / 2}}{(t+|x-y|)^{1+2 \nu}} \chi_{\left\{\frac{x}{2}<y<M x\right\}}+C_{2}(x)(t \vee 1)^{2 \nu} \Phi(y),
$$

where $C_{1}(x)=(1+x)^{2 \nu} e^{\frac{x^{2}}{2}}$ and $C_{2}(x)=[\log (e+x)]^{1+\nu}(1+x)^{\left|\mu+\frac{1}{2}\right|} e^{\frac{x^{2}}{2}} /\langle x\rangle^{\alpha+\frac{3}{2}}$.

If we consider, for fixed $M>1$, the local maximal function in $\mathbb{R}_{+}$

$$
\mathcal{M}_{M}^{\mathrm{loc}} f(x):=\sup _{t>0} \frac{1}{\left|I_{t}(x)\right|} \int_{I_{t}(x)}|f(y)| \chi_{\left\{\frac{x}{2}<y<M x\right\}} d y,
$$

then we may express (5.8) as follows.

Corollary 5.3. Let $t_{0}>0$ be fixed. Then there is some $M>1$ such that

$$
P_{t_{0}}^{*} f(x) \lesssim C_{1}(x) \mathcal{M}_{M}^{\text {loc }}\left(f e^{-\frac{y^{2}}{2}}\right)(x)+C_{2}(x)\|f\|_{L^{1}(\Phi)}, \quad x \in \mathbb{R}_{+},
$$

with $C_{1}(x)$ and $C_{2}(x)$ as in Proposition 5.2.

This is the key estimate from which we shall deduce the theorems claimed in $\S 1$. The interested reader may wish to skip the technical proofs of the propositions in the next subsections and pass directly to $₫ 6$ for the proof of the theorems.

5.1. Estimates from below for $B_{t}(x, y)$. Recall that

$$
B_{t}(x, y) \approx t^{2 \nu}(x y)^{\alpha+\frac{1}{2}} e^{\frac{x^{2}-y^{2}}{2}} \int_{0}^{r_{0}(x y)} \frac{r^{\alpha+\mu+1}}{(1-r)^{\alpha+1}\left(\ln \frac{1}{r}\right)^{1+\nu}} e^{-\frac{t^{2}}{2 \ln \frac{1}{r}}} e^{-\frac{(x-r y)^{2}}{1-r^{2}}} \frac{d r}{r} .
$$

The lower bound in Proposition 5.1 will be obtained by just looking at this integral.

Lemma 5.4. For fixed $t, x>0$ it holds

$$
B_{t}(x, y) \geq c_{1}(t, x) \Phi(y), \quad \forall y \in \mathbb{R}_{+},
$$

for a suitable function $c_{1}(t, x)>0$. 
Proof: We first look at $y<x \wedge \frac{1}{x}$. Then $x y<1$, and hence $r_{0}(x y)>1 / 2$. So, we can estimate $B_{t}(x, y)$ by an integral over $0<r<\frac{1}{2}$, which disregarding irrelevant terms becomes

$$
B_{t}(x, y) \gtrsim t^{2 \nu}(x y)^{\alpha+\frac{1}{2}} e^{\frac{x^{2}-y^{2}}{2}} \int_{0}^{\frac{1}{2}} \frac{r^{\alpha+\mu+1}}{\left(\ln \frac{1}{r}\right)^{1+\nu}} e^{-\frac{t^{2}}{2 \ln \frac{1}{r}}} e^{-\frac{(x-r y)^{2}}{1-r^{2}}} \frac{d r}{r} .
$$

We can get rid of the first two exponentials using

$$
e^{\frac{x^{2}-y^{2}}{2}} \geq 1 \quad(\text { since } y \leq x) \quad \text { and } \quad e^{-\frac{t^{2}}{2 \ln \frac{1}{r}}} \geq e^{-\frac{t^{2}}{2 \ln 2}} \quad\left(\text { since } r \leq \frac{1}{2}\right) .
$$

For the last exponential notice that $0<x-r y \leq x$, and hence $e^{-\frac{(x-r y)^{2}}{1-r^{2}}} \geq e^{-\frac{4}{3} x^{2}}$. This leaves a convergent integral in $r$, so we conclude that

$$
B_{t}(x, y) \gtrsim c_{1}(t, x)\langle y\rangle^{\alpha+\frac{1}{2}},
$$

with $c_{1}(t, x)=t^{2 \nu} x^{\alpha+\frac{1}{2}} e^{-\frac{t^{2}}{2 \ln 2}} e^{-\frac{4}{3} x^{2}}$. Notice that $y \leq 1$ in this range, so we find the required expression for $\Phi(y)$.

Suppose now that $y \geq x \vee \frac{1}{x}$. Then $x y \geq 1$, and hence $r_{0}(x y)=\frac{1}{2 x y} \leq 1 / 2$. Arguing as before we can estimate $B_{t}(x, y)$ by

$$
B_{t}(x, y) \gtrsim t^{2 \nu}(x y)^{\alpha+\frac{1}{2}} e^{-\frac{y^{2}}{2}} e^{-\frac{t^{2}}{2 \ln 2}} \int_{0}^{\frac{1}{2 x y}} \frac{r^{\alpha+\mu+1}}{\left(\ln \frac{1}{r}\right)^{1+\nu}} e^{-\frac{(x-r y)^{2}}{1-r^{2}}} \frac{d r}{r} .
$$

This time we get rid of the exponential inside the integral using

$$
|x-r y| \leq x+r y \leq x+\frac{1}{2 x} \quad\left(\text { since } r \leq \frac{1}{2 x y}\right),
$$

which implies $e^{-\frac{(x-r y)^{2}}{1-r^{2}}} \geq e^{-\frac{4}{3}\left(x+\frac{1}{x}\right)^{2}}$. We can easily compute the integral

$$
\int_{0}^{\frac{1}{2 x y}} \frac{r^{\alpha+\mu+1}}{\left(\ln \frac{1}{r}\right)^{1+\nu}} \frac{d r}{r} \approx \frac{1}{(x y)^{\alpha+\mu+1}[\log 2 x y]^{1+\nu}}, \quad \text { if } \alpha+\mu+1>0,
$$

with the right hand side becoming $1 /[\log 2 x y]^{\nu}$ in the extreme case $\alpha+1+\mu=0$. Since $y>\max \{x, 1\}$, note that

$$
\log (2 x y) \leq \log \left(2 y^{2}\right) \approx \log (y+e) .
$$

Thus, combining all the previous estimates we conclude that

$$
B_{t}(x, y) \gtrsim c_{1}(t, x) \frac{e^{-\frac{y^{2}}{2}}}{y^{\mu+\frac{1}{2}}[\log (y+e)]^{1+\nu}},
$$

which, since $y \geq 1$, is the required expression for $\Phi(y)$ (with the usual agreement when $\mu+\alpha+1=0)$. In this part we have set $c_{1}(t, x)=t^{2 \nu} e^{-\frac{t^{2}}{2 \ln 2}} x^{-\left(\mu+\frac{1}{2}\right)} e^{-\frac{4}{3}\left(x+\frac{1}{x}\right)^{2}}$.

Finally, since the function $y \mapsto P_{t}(x, y) / \Phi(y)$ is continuous and positive, it is also bounded from below by some $c_{1}(t, x)$ when $y$ belongs to the compact set $\left[x \wedge \frac{1}{x}, x \vee \frac{1}{x}\right]$. 
5.2. Estimates from above for $B_{t}(x, y)$. The next lemma, combined with the previous one, shows that for fixed $t$ and $x$, the function $B_{t}(x, y)$ essentially behaves like $\Phi(y)$.

Lemma 5.5. For fixed $t, x>0$ it holds

$$
B_{t}(x, y) \lesssim c(x) \max \left\{t^{2 \nu}, 1\right\} \Phi(y), \quad \forall y \in \mathbb{R}_{+},
$$

with $c(x)=1 /\langle x\rangle^{\alpha+\frac{3}{2}}$.

Proof: We first notice that the two exponential terms in (5.3) can be written as

$$
e^{-\frac{(x-r y)^{2}}{1-r^{2}}} e^{\frac{x^{2}-y^{2}}{2}}=e^{-\frac{1+r^{2}}{1-r^{2}} \frac{x^{2}+y^{2}}{2}} e^{\frac{2 r x y}{1-r^{2}}} \lesssim e^{-\frac{x^{2}+y^{2}}{2}},
$$

since $\frac{1+r^{2}}{1-r^{2}} \geq 1$ and in the region of integration of $B_{t}(x, y)$ the exponent $z=\frac{2 r x y}{1-r^{2}} \lesssim 1$. We now separate cases.

(i) Case $x y \geq 1$ : then $r_{0}(x y)=\frac{1}{2 x y} \leq \frac{1}{2}$ and

$$
B_{t}(x, y) \lesssim t^{2 \nu}(x y)^{\alpha+\frac{1}{2}} e^{-\frac{x^{2}+y^{2}}{2}} \int_{0}^{\frac{1}{2 x y}} \frac{r^{\alpha+\mu+1}}{\left(\ln \frac{1}{r}\right)^{\nu+1}} \frac{d r}{r} .
$$

The last integral is approximately given by

$$
\int_{0}^{\frac{1}{2 x y}} \frac{r^{\alpha+\mu+1}}{\left(\ln \frac{1}{r}\right)^{\nu+1}} \frac{d r}{r} \approx\left(\frac{1}{x y}\right)^{\alpha+\mu+1} \frac{1}{[\log (2 x y)]^{1+\nu}}
$$

(with the usual convention when $\alpha+\mu+1=0$ of reducing the log by one power). This is a good estimate if we assume that $x \geq 1 / 2$, since we may use

$$
\log (2 x y) \gtrsim \log (y \vee 2) \approx \log (y+e),
$$

and overall obtain

$$
B_{t}(x, y) \lesssim t^{2 \nu} x^{-\left(\mu+\frac{1}{2}\right)} e^{-\frac{x^{2}}{2}} \frac{y^{\alpha+\frac{1}{2}} e^{-y^{2} / 2}}{y^{\alpha+\mu+1}[\log (y+e)]^{1+\nu}} \lesssim t^{2 \nu} \Phi(y) .
$$

When $x \leq 1 / 2$, we need a refinement to obtain the $c(x)$ in the statement of the lemma. We split the integral defining $B_{t}(x, y)$ as

$$
B_{t}(x, y)=\int_{0}^{\frac{2 x}{y}} \cdots+\int_{\frac{2 x}{y}}^{r_{0}(x y)} \cdots=I+I I,
$$

noticing that the partition point $\frac{2 x}{y} \leq r_{0}(x y)=\frac{1}{2 x y}$. Since $x \leq \frac{1}{2}$ and $x y \geq 1$ we also have $y \geq 2$. Now, the first integral can be bound as above by

$$
\begin{aligned}
I & \lesssim t^{2 \nu}(x y)^{\alpha+\frac{1}{2}} e^{-\frac{x^{2}+y^{2}}{2}}\left(\frac{x}{y}\right)^{\alpha+\mu+1} \frac{1}{\left[\log \left(\frac{y}{2 x}\right)\right]^{1+\nu}} \\
& \lesssim t^{2 \nu}\langle x\rangle^{\alpha+\frac{1}{2}} x^{\alpha+\mu+1} e^{-\frac{x^{2}}{2}} \frac{e^{-y^{2} / 2}}{y^{\mu+\frac{1}{2}}[\log y]^{1+\nu}} \lesssim t^{2 \nu}\langle x\rangle^{\alpha+\frac{1}{2}} \Phi(y) .
\end{aligned}
$$

since in this range $y \geq 2$. This implies the stated estimate because $\langle x\rangle^{\alpha+\frac{1}{2}} \leq 1 /\langle x\rangle^{\alpha+\frac{3}{2}}=$ $c(x)$. To handle $I I$ we need a different bound for the exponentials in (5.13), noticing that

$$
r>\frac{2 x}{y} \Rightarrow|x-r y|=r y-x \geq \frac{r y}{2} \Rightarrow e^{-\frac{(x-r y)^{2}}{1-r^{2}}} \leq e^{-\frac{r^{2} y^{2}}{4}} .
$$


Thus

$$
I I \lesssim t^{2 \nu}(x y)^{\alpha+\frac{1}{2}} e^{\frac{x^{2}-y^{2}}{2}} \int_{\frac{2 x}{y}}^{\frac{1}{2}} \frac{r^{\alpha+\mu+1} e^{-\frac{(r y)^{2}}{4}}}{\left(\ln \frac{1}{r}\right)^{\nu+1}} \frac{d r}{r} .
$$

Changing variables $r y=u$, the latter integral can be estimated by

$$
y^{-(\alpha+\mu+1)} \int_{0}^{\frac{y}{2}} \frac{u^{\alpha+\mu+1} e^{-\frac{u^{2}}{4}}}{\left(\ln \frac{y}{u}\right)^{\nu+1}} \frac{d u}{u} \approx \frac{1}{y^{\alpha+\mu+1}[\log y]^{1+\nu}}
$$

since the major contribution happens when $u \approx 1$ (with the usual convention of reducing a $\log$ power if $\alpha+\mu+1=0$ ). Inserting this into (5.17) (and using $y \geq 2$ and $x \leq 1 / 2$ ) we obtain once again

$$
I I \lesssim t^{2 \nu}\langle x\rangle^{\alpha+\frac{1}{2}} \Phi(y)
$$

This concludes the proof of the case $x y \geq 1$.

(ii) Case $x y \leq 1$ : this time $r_{0}(x y)=1-\frac{x y}{2} \geq \frac{1}{2}$, so we may split

$$
B_{t}(x, y)=\int_{0}^{\frac{1}{2}} \cdots+\int_{\frac{1}{2}}^{r_{0}(x y)} \cdots=B_{1}+B_{2}
$$

The first term can be handled essentially as in the previous case. Namely, if $y \leq 2$ we use a similar bound to (5.14)

$$
B_{1} \lesssim t^{2 \nu}(x y)^{\alpha+\frac{1}{2}} e^{-\frac{x^{2}+y^{2}}{2}} \int_{0}^{\frac{1}{2}} \frac{r^{\alpha+\mu+1}}{\left(\ln \frac{1}{r}\right)^{\nu+1}} \frac{d r}{r} \approx t^{2 \nu} x^{\alpha+\frac{1}{2}} e^{-\frac{x^{2}}{2}}\langle y\rangle^{\alpha+\frac{1}{2}} \lesssim t^{2 \nu}\langle x\rangle^{\alpha+\frac{1}{2}} \Phi(y) .
$$

If $y \geq 2$, then $x \leq \frac{1}{2}$ and $\frac{2 x}{y} \leq \frac{1}{2}$, so we may split

$$
B_{1} \leq \int_{0}^{\frac{2 x}{y}} \cdots+\int_{\frac{2 x}{y}}^{\frac{1}{2}} \cdots
$$

and exactly the same computations we used in (5.15), give us the bound

$$
B_{1} \lesssim t^{2 \nu}\langle x\rangle^{\alpha+\frac{1}{2}} \Phi(y)
$$

Thus we are left with the integral corresponding to $B_{2}$, that is the range $\frac{1}{2}<r<1-\frac{x y}{2}$. First of all, observe that

$$
\ln \frac{1}{r} \approx 1-r, \quad r \in[1 / 2,1] \quad \Rightarrow \quad e^{-\frac{t^{2}}{2 \ln \frac{1}{r}}} \leq e^{-\frac{c t^{2}}{1-r}}
$$

for a suitable $c>0$. Next, we need once again more precise bounds for the exponentials in (5.13). We claim that, if $r \in[1 / 2,1]$ then

$$
e^{-\frac{1+r^{2}}{1-r^{2}} \frac{x^{2}+y^{2}}{2}} \leq e^{-\gamma \frac{x^{2}+y^{2}}{1-r}} e^{-(1+\gamma) \frac{x^{2}+y^{2}}{2}}
$$


for a small constant $\gamma>0$. This is easily obtained using the fact that $\frac{1+r^{2}}{1-r^{2}} \geq \frac{5}{3}$ in this interval. With these exponential bounds we can control the integral $B_{2}$ as follows

$$
\begin{aligned}
B_{2} & \lesssim t^{2 \nu}(x y)^{\alpha+\frac{1}{2}} e^{-(1+\gamma) \frac{x^{2}+y^{2}}{2}} \int_{1 / 2}^{1-\frac{x y}{2}} \frac{e^{-\frac{c t^{2}+\gamma\left(x^{2}+y^{2}\right)}{1-r}}}{(1-r)^{\alpha+\nu+1}} \frac{d r}{1-r} \\
& \lesssim \frac{t^{2 \nu}(x y)^{\alpha+\frac{1}{2}}}{\left[t^{2}+x^{2}+y^{2}\right]^{\alpha+\nu+1}} e^{-(1+\gamma) \frac{x^{2}+y^{2}}{2}} \int_{0}^{\infty} e^{-u} u^{\alpha+\nu+1} \frac{d u}{u}
\end{aligned}
$$

after changing variables $u=\left[c t^{2}+\gamma\left(x^{2}+y^{2}\right)\right] /(1-r)$. The last integral is a finite constant (because $\alpha+\nu+1>0$ ), so we observe three possible cases:

(1) if $y \geq 1$, we can disregard the denominator and obtain

$$
B_{2} \lesssim t^{2 \nu}(x y)^{\alpha+\frac{1}{2}} e^{-(1+\gamma) \frac{y^{2}}{2}} \lesssim t^{2 \nu}\langle x\rangle^{\alpha+\frac{1}{2}} \Phi(y),
$$

since the exponential decay in $y$ is actually better than $\Phi(y)$ (and also $x \leq 1$ ).

(2) if $y \leq 1$ and $\max \{x, t\} \geq 1$, we can also disregard the denominator and obtain

$$
B_{2} \lesssim t^{2 \nu}\langle y\rangle^{\alpha+\frac{1}{2}} x^{\alpha+\frac{1}{2}} e^{-\frac{x^{2}}{2}} \lesssim t^{2 \nu}\langle x\rangle^{\alpha+\frac{1}{2}}\langle y\rangle^{\alpha+\frac{1}{2}}
$$

(3) if all $y, t, x \leq 1$, we bound the denominator in the two obvious ways to obtain

$$
B_{2} \lesssim \frac{t^{2 \nu}(x y)^{\alpha+\frac{1}{2}}}{t^{2 \nu} x^{2(\alpha+1)}}=\frac{\langle y\rangle^{\alpha+\frac{1}{2}}}{\langle x\rangle^{\alpha+\frac{3}{2}}},
$$

which is precisely the upper bound stated in (5.12). Observe that when $x \rightarrow 0$ this piece gives the largest contribution to $B_{t}(x, y)$.

Remark 5.6. It is also possible to obtain a bound

$$
B_{t}(x, y) \lesssim c^{\prime}(x) t^{2 \nu} \Phi(y),
$$

with perhaps a worse function $c^{\prime}(x)$, but without the loss produced by $\max \left\{1, t^{2 \nu}\right\}$. This loss appeared when $t, x, y \leq 1$ in (5.19) above. Looking at (5.18) we may replace that bound by

$$
B_{2} \lesssim \frac{t^{2 \nu}(x y)^{\alpha+\frac{1}{2}}}{x^{2(\alpha+\nu+1)}}
$$

which implies (5.20) with $c^{\prime}(x)=1 / l x^{\alpha+\frac{3}{2}+2 \nu}$. This estimate will also be useful later.

5.3. Upper estimates for $A_{t}(x, y)$ : integrals over $r \leq 1 / 2$. Recall that

$$
A_{t}(x, y) \approx t^{2 \nu} e^{\frac{x^{2}-y^{2}}{2}} \int_{r_{0}(x y)}^{1} \frac{r^{\mu+\frac{1}{2}}}{\sqrt{1-r}\left(\ln \frac{1}{r}\right)^{1+\nu}} e^{-\frac{t^{2}}{2 \ln \frac{1}{r}}} e^{-\frac{(x-r y)^{2}}{1-r^{2}}} \frac{d r}{r} .
$$

When $x y \geq 1$ we have $r_{0}(x y)=\frac{1}{2 x y} \leq \frac{1}{2}$, so we can write

$$
A_{t}(x, y)=\int_{r_{0}(x y)}^{\frac{1}{2}} \cdots+\int_{\frac{1}{2}}^{1} \cdots=A 1+A 2 .
$$

In this section we shall prove the following estimate for $A 1$. 
Lemma 5.7. If $x y \geq 1$, then

$$
A 1 \lesssim t^{2 \nu} e^{\frac{x^{2}-y^{2}}{2}} \int_{\frac{1}{2 x y}}^{\frac{1}{2}} \frac{r^{\mu+\frac{1}{2}}}{\left(\ln \frac{1}{r}\right)^{1+\nu}} e^{-\frac{(x-r y)^{2}}{1-r^{2}}} \frac{d r}{r} \lesssim c(x) t^{2 \nu} \Phi(y),
$$

where $c(x)=[\log (e+x)]^{\nu+1}(1+x)^{\left|\mu+\frac{1}{2}\right|} \exp \left(x^{2} / 2\right)$.

Proof: We shall distinguish cases

(i) Case $y \leq 4 x$. In this region we essentially disregard the exponential term $e^{-\frac{(x-r y)^{2}}{1-r^{2}}}$ inside the integral, and directly estimate

$$
A 1 \lesssim t^{2 \nu} e^{\frac{x^{2}-y^{2}}{2}} \int_{\frac{1}{2 x y}}^{\frac{1}{2}} \frac{r^{\mu+\frac{1}{2}}}{\left(\ln \frac{1}{r}\right)^{1+\nu}} \frac{d r}{r} .
$$

Notice however that when $y \leq x$ the exponential produces an additional gain, due to

$$
r y \leq \frac{x}{2} \Rightarrow|x-r y| \geq \frac{x}{2} \Rightarrow e^{-\frac{(x-r y)^{2}}{1-r^{2}}} \leq e^{-\frac{x^{2}}{4}} .
$$

This will play a role later in evaluating the constant $c(x)$. We now evaluate the integral in (5.23), depending on the sign of $\mu+\frac{1}{2}$.

(1) If $\mu+\frac{1}{2} \geq 0$ the integral is bounded by a constant, and hence

$$
A 1 \lesssim t^{2 \nu} e^{\frac{x^{2}-y^{2}}{2}}
$$

We shall enlarge this value to match (5.22) as follows. Since $x y \geq 1$, in this range we have $x \geq 1 / 2$. So if $1 \leq y \leq 4 x$ we may use

$$
1 \lesssim \frac{(1+x)^{\mu+\frac{1}{2}}[\log (e+x)]^{\nu+1}}{(1+y)^{\mu+\frac{1}{2}}[\log (e+y)]^{\nu+1}}
$$

If $\frac{1}{x} \leq y \leq 1$, we use instead

$$
1 \lesssim \max \left\{x^{\alpha+\frac{1}{2}}, 1\right\}\langle y\rangle^{\alpha+\frac{1}{2}}
$$

which in this region can be combined with the extra exponential in factor in (5.24).

In both cases we obtain $A 1 \lesssim t^{2 \nu} c(x) \Phi(y)$, as wished.

(2) If $\mu+\frac{1}{2}<0$ the integral diverges near 0 , but we still obtain

$$
\int_{\frac{1}{2 x y}}^{\frac{1}{2}} \frac{r^{\mu+\frac{1}{2}}}{\left(\ln \frac{1}{r}\right)^{1+\nu}} \frac{d r}{r} \lesssim \frac{1}{(x y)^{\mu+\frac{1}{2}}(\log 2 x y)^{\nu+1}} .
$$

Thus, using the inequality $\log (2 x y) \gtrsim \max \{\log y, \log 2\}$ we arrive at

$$
\begin{aligned}
A 1 & \lesssim t^{2 \nu} e^{\frac{x^{2}-y^{2}}{2}} \frac{(1+x)^{\left|\mu+\frac{1}{2}\right|}}{(1+y)^{\mu+\frac{1}{2}}[\log (e+y)]^{\nu+1}} \leq t^{2 \nu} c(x) \Phi(y), \quad \text { if } 1 \leq y \leq 4 x \\
& \lesssim t^{2 \nu} \frac{e^{\frac{x^{2}}{4}} y^{\alpha+\frac{1}{2}}}{x^{\mu+\frac{1}{2}} y^{\mu+\alpha+1}} \leq t^{2 \nu} e^{\frac{x^{2}}{4}} x^{\alpha+\frac{1}{2}}\langle y\rangle^{\alpha+\frac{1}{2}} \leq t^{2 \nu} c(x) \Phi(y), \quad \text { if } \frac{1}{x} \leq y \leq 1,
\end{aligned}
$$

using in the last case the additional exponential gain in (5.24). 
(ii) Case $y \geq 4 x$. This is the same as $\frac{2 x}{y} \leq \frac{1}{2}$, and remember from (5.16) that when $r \in\left[\frac{2 x}{y}, \frac{1}{2}\right]$ a better bound for the exponential is available, namely

$$
e^{-\frac{(x-r y)^{2}}{1-r^{2}}} \leq e^{-\frac{r^{2} y^{2}}{4}}
$$

Thus we may consider two subcases, depending on whether $\frac{2 x}{y}$ is above or below $r_{0}(x y)$.

- Subcase $\frac{2 x}{y} \leq r_{0}(x y)=\frac{1}{2 x y} \leq \frac{1}{2}$. Using (5.25) we obtain

$$
\begin{aligned}
A 1 & \lesssim t^{2 \nu} e^{\frac{x^{2}-y^{2}}{2}} \int_{\frac{1}{2 x y}}^{\frac{1}{2}} \frac{r^{\mu+\frac{1}{2}} e^{-\frac{(r y)^{2}}{4}}}{\left(\ln \frac{1}{r}\right)^{1+\nu}} \frac{d r}{r} \\
(r y=u) & =\frac{t^{2 \nu} e^{\frac{x^{2}-y^{2}}{2}}}{y^{\mu+\frac{1}{2}}} \int_{\frac{1}{2 x}}^{\infty} \frac{u^{\mu+\frac{1}{2}} e^{-\frac{u^{2}}{4}}}{\left(\ln \frac{y}{u}\right)^{1+\nu}} \frac{d u}{u}
\end{aligned}
$$

Observe that $x \leq \frac{1}{2}$ (and $y \geq 2$ ), so the latter integral reaches its major contribution at $u=\frac{1}{2 x}$

$$
\int_{\frac{1}{2 x}}^{\infty} \frac{u^{\mu+\frac{1}{2}} e^{-\frac{u^{2}}{4}}}{\left(\ln \frac{y}{u}\right)^{1+\nu}} \frac{d u}{u} \lesssim \frac{(1 / x)^{\mu-\frac{1}{2}} e^{-c / x^{2}}}{(\log 2 x y)^{1+\nu}} \lesssim \frac{1}{(\log y)^{1+\nu}}
$$

using in the last step the elementary bound of logarithms

$$
\log (2 x y) \gtrsim \frac{\log (y+e)}{\log \left(\frac{1}{x}+e\right)}, \quad \text { if } y \geq \max \{1,1 / x\}
$$

(see e.g. [4, Lemma 5.1]). Thus we conclude that

$$
A 1 \lesssim t^{2 \nu} e^{\frac{x^{2}}{2}} \Phi(y)
$$

- Subcase $r_{0}(x y)<\frac{2 x}{y} \leq \frac{1}{2}$. Here we split

$$
A 1=\int_{\frac{2 x}{y}}^{\frac{1}{2}} \cdots+\int_{r_{0}(x y)}^{\frac{2 x}{y}} \cdots=I+I I .
$$

The first term is similar to the previous subcase, except that now $x>\frac{1}{2}$ (and $y \geq 4 x \geq 2$ )

$$
I \lesssim \frac{t^{2 \nu} e^{\frac{x^{2}-y^{2}}{2}}}{y^{\mu+\frac{1}{2}}} \int_{\frac{x}{2}}^{\infty} \frac{u^{\mu+\frac{1}{2}} e^{-\frac{u^{2}}{4}}}{\left(\ln \frac{y}{u}\right)^{1+\nu}} \frac{d u}{u}
$$

and the last integral is bounded by a constant times

$$
\frac{x^{\mu-\frac{1}{2}} e^{-c x^{2}}}{\left(\ln \frac{2 y}{x}\right)^{1+\nu}} \lesssim x^{\mu-\frac{1}{2}} e^{-c x^{2}} \frac{[\log (e+x)]^{1+\nu}}{[\log (e+y)]^{1+\nu}} \lesssim \frac{1}{[\log (e+y)]^{1+\nu}} .
$$

Finally, we consider II. Here there is no exponential gain, and similarly to (5.23) we have

$$
I I \lesssim t^{2 \nu} e^{\frac{x^{2}-y^{2}}{2}} \int_{\frac{1}{2 x y}}^{\frac{2 x}{y}} \frac{r^{\mu+\frac{1}{2}}}{\left(\ln \frac{1}{r}\right)^{1+\nu}} \frac{d r}{r}=\frac{t^{2 \nu} e^{\frac{x^{2}-y^{2}}{2}}}{y^{\mu+\frac{1}{2}}} \int_{\frac{1}{2 x}}^{2 x} \frac{u^{\mu+\frac{1}{2}}}{\left(\ln \frac{y}{u}\right)^{1+\nu}} \frac{d u}{u} .
$$

Now, the last integral can easily be analyzed (depending on the sign of $\mu+\frac{1}{2}$ ) to obtain

$$
\int_{\frac{1}{2 x}}^{2 x} \frac{u^{\mu+\frac{1}{2}}}{\left(\ln \frac{y}{u}\right)^{1+\nu}} \frac{d u}{u} \lesssim \frac{x^{\left|\mu+\frac{1}{2}\right|}[\log (x+e)]^{1+\nu}}{[\log (y+e)]^{1+\nu}} .
$$


Thus, overall we conclude that in this subcase

$$
A 1 \lesssim I+I I \lesssim t^{2 \nu}(1+x)^{\left|\mu+\frac{1}{2}\right|}[\log (x+e)]^{1+\nu} e^{\frac{x^{2}}{2}} \Phi(y) .
$$

5.4. Upper estimates for $A_{t}(x, y)$ when $y \leq x / 2$ or $y \geq M x$. In view of the previous subsection, it only remains to estimate

$$
\begin{aligned}
A 2 & \approx t^{2 \nu} e^{\frac{x^{2}-y^{2}}{2}} \int_{\max \left\{r_{0}(x y), \frac{1}{2}\right\}}^{1} \frac{e^{-\frac{t^{2}}{2 \ln \frac{1}{r}}}}{\sqrt{1-r}\left(\ln \frac{1}{r}\right)^{1+\nu}} e^{-\frac{(x-r y)^{2}}{1-r^{2}}} d r \\
& \lesssim t^{2 \nu} e^{\frac{x^{2}-y^{2}}{2}} \int_{\max \left\{1-\frac{x y}{2}, \frac{1}{2}\right\}}^{1} \frac{e^{-\frac{c t^{2}}{1-r}}}{(1-r)^{\nu+\frac{3}{2}}} e^{-\frac{(x-r y)^{2}}{1-r^{2}}} d r
\end{aligned}
$$

noticing that $\ln \frac{1}{r} \approx 1-r$ when $r \in\left[\frac{1}{2}, 1\right]$. In this region, however, it is more convenient to use the write-up for the heat kernel in (5.4), in terms of the parameter $s$. This gives a more reasonable expression for the exponentials, namely

$$
e^{\frac{x^{2}-y^{2}}{2}} e^{-\frac{(x-r y)^{2}}{1-r^{2}}}=e^{-\frac{1}{4}\left[\frac{(x-y)^{2}}{s}+s(x+y)^{2}\right]} .
$$

Since the parameters $r$ and $s$ are related by $s=\frac{1-r}{1+r}$ (or $r=\frac{1-s}{1+s}$ ), either from (5.4) or directly from (5.26)

$$
A 2 \lesssim t^{2 \nu} \int_{0}^{\min \left\{\frac{1}{3}, \frac{x y}{3}\right\}} \frac{e^{-\frac{c t^{2}}{s}} e^{-\frac{1}{4}\left[\frac{(x-y)^{2}}{s}+s(x+y)^{2}\right]}}{s^{\nu+\frac{3}{2}}} d s
$$

after perhaps slightly enlarging the range of integration. Our first result shows that when $y$ is far from $x$ this can also be controlled by the function $\Phi(y)$.

Lemma 5.8. There exists $M>1$ such that, if $y \leq \frac{x}{2}$ or $y \geq M x$, then

$$
A 2 \lesssim t^{2 \nu} \int_{0}^{\min \left\{\frac{1}{3}, \frac{x y}{3}\right\}} \frac{e^{-\frac{c t^{2}}{s}} e^{-\frac{1}{4}\left[\frac{(x-y)^{2}}{s}+s(x+y)^{2}\right]}}{s^{\nu+\frac{3}{2}}} d s \lesssim c(x) \max \left\{t^{2 \nu}, 1\right\} \Phi(y),
$$

with $c(x)=1 /\langle x\rangle^{\alpha+\frac{3}{2}}$.

PRoof: We claim that, in the assumed range of $x$ and $y$, there is some $\gamma>0$ such that

$$
A 2 \lesssim t^{2 \nu} e^{-\left(\frac{1}{2}+\gamma\right) y^{2}} \int_{0}^{\min \left\{\frac{1}{3}, \frac{x y}{3}\right\}} e^{-\gamma \frac{t^{2}+(x-y)^{2}}{s}} s^{-\left(\nu+\frac{3}{2}\right)} d s
$$

This is just a bound of the exponentials. Indeed, if we distinguish the two cases

(1) case $y \geq M x$ : this implies $|y-x| \geq\left(1-\frac{1}{M}\right) y$, so for any $\eta<1$ we have

$$
e^{-\frac{c t^{2}}{s}} e^{-\frac{1}{4}\left[\frac{(x-y)^{2}}{s}+s(x+y)^{2}\right]} \leq e^{-\frac{c t^{2}+\frac{\eta}{4}(x-y)^{2}}{s}} e^{-\frac{1-\eta}{4}\left(\frac{M-1}{M}\right)^{2}\left(\frac{1}{s}+s\right) y^{2}}
$$

which implies the required assertion using that $\frac{1}{s}+s \geq \frac{10}{3}$, when $s \in\left(0, \frac{1}{3}\right)$, and choosing $M$ sufficiently large and $\eta$ sufficiently small. 
(2) case $y \leq x / 2$ : this time $|x-y| \geq \frac{x}{2} \geq y$, so we have

$$
e^{-\frac{c t^{2}}{s}} e^{-\frac{1}{4}\left[\frac{(x-y)^{2}}{s}+s(x+y)^{2}\right]} \leq e^{-\frac{c t^{2}+\frac{\eta}{4}(x-y)^{2}}{s}} e^{-\frac{1-\eta}{4}\left(\frac{1}{s}+s\right) y^{2}}
$$

which again implies the assertion using $\frac{1}{s}+s \geq \frac{10}{3}$ and choosing $\eta$ sufficiently small. Thus (5.28) is proven, and we may change variables $\left[t^{2}+(x-y)^{2}\right] / s=u$ to obtain

$$
\begin{aligned}
A 2 & \lesssim \frac{t^{2 \nu} e^{-\left(\frac{1}{2}+\gamma\right) y^{2}}}{\left[t^{2}+(x-y)^{2}\right]^{\nu+\frac{1}{2}}} \int_{3 \gamma\left[t^{2}+(x-y)^{2}\right] \max \left\{1, \frac{1}{x y}\right\}}^{\infty} u^{\nu+\frac{1}{2}} e^{-u} \frac{d u}{u} \\
& \lesssim \frac{t^{2 \nu} e^{-\left(\frac{1}{2}+\gamma\right) y^{2}}}{(t+x+y)^{2 \nu+1}} \int_{\gamma^{\prime}\left[x^{2}+y^{2}\right] \max \left\{1, \frac{1}{x y}\right\}}^{\infty} u^{\nu+\frac{1}{2}} e^{-u} \frac{d u}{u},
\end{aligned}
$$

since in the selected range of $x, y$ we have $|x-y| \gtrsim x+y$. To finish the proof we must distinguish some cases.

Case $y \geq 1$ : then bounding the denominator and the integral in (5.30) by a constant we immediately see that

$$
A 2 \lesssim t^{2 \nu} e^{-\left(\frac{1}{2}+\gamma\right) y^{2}} \lesssim t^{2 \nu} \Phi(y)
$$

since the exponential has a better decay.

Case $y \leq 1$ and $y \geq M x$ : we again bound the integral by a constant and estimate the fraction in (5.30) as follows

$$
A 2 \lesssim \frac{t^{2 \nu}}{t^{2 \nu} y}=\frac{\langle y\rangle^{\alpha+\frac{1}{2}}}{y^{\alpha+\frac{3}{2}}} \leq c_{M} \frac{\langle y\rangle^{\alpha+\frac{1}{2}}}{\langle x\rangle^{\alpha+\frac{3}{2}}} .
$$

Case $y \leq 1$ and $y \leq \frac{x}{2}$ : this is a relevant case, since the integral in (5.30) plays actually a role. To compute the integral we must distinguish the two subcases

(1) If $x y \leq 1$, then since also $\frac{x}{y} \geq 2$,

$$
\begin{aligned}
A 2 & \lesssim \frac{t^{2 \nu}}{t^{2 \nu} x} \int_{\gamma^{\prime} \frac{x}{y}}^{\infty} u^{\nu-\frac{1}{2}} e^{-u} d u \approx \frac{1}{x}\left(\frac{x}{y}\right)^{\nu-\frac{1}{2}} e^{-c \frac{x}{y}} \\
& \lesssim \frac{1}{x}\left(\frac{y}{x}\right)^{\alpha+\frac{1}{2}} \leq \frac{\langle y\rangle^{\alpha+\frac{1}{2}}}{\langle x\rangle^{\alpha+\frac{3}{2}}} .
\end{aligned}
$$

(2) If $x y \geq 1$, then we have $x \geq \frac{1}{y} \geq 1$ and

$$
A 2 \lesssim \frac{t^{2 \nu}}{x^{1+2 \nu}} \int_{\gamma^{\prime} x^{2}}^{\infty} u^{\nu-\frac{1}{2}} e^{-u} d u \lesssim t^{2 \nu} x^{-2} e^{-c x^{2}}
$$

Now, since $\frac{1}{x} \leq y \leq 1$ we can insert the estimate

$$
1 \lesssim\langle y\rangle^{\alpha+\frac{1}{2}} \max \left\{x^{\alpha+\frac{1}{2}}, 1\right\},
$$

to obtain $A 2 \lesssim t^{2 \nu}\langle y\rangle^{\alpha+\frac{1}{2}}$. 
Remark 5.9. As mentioned earlier in Remark 5.6, here it is also possible to obtain a bound

$$
A 2 \lesssim c^{\prime}(x) t^{2 \nu} \Phi(y)
$$

with $c^{\prime}(x)=1 / l x^{\alpha+\frac{3}{2}+2 \nu}$. The loss produced by $\max \left\{1, t^{2 \nu}\right\}$ can be corrected in (5.31) and (5.32) by replacing the factor $t^{2 \nu}$ in the denominator by $x^{2 \nu}$, as one readily notices from (5.30). As mentioned before, this estimate will play a role later.

5.5. Upper estimates for $A_{t}(x, y)$ in the local part $\frac{x}{2}<y<M x$. As in the previous subsection, our starting point is the formula (5.27), which we must estimate in the local region $\frac{x}{2}<y<M x$. A sufficient bound for us is stated in the next lemma.

Lemma 5.10. If $\frac{x}{2}<y<M x$, then

$$
t^{2 \nu} \int_{0}^{\min \left\{\frac{1}{3}, \frac{x y}{3}\right\}} \frac{e^{-\frac{c t^{2}}{s}-\frac{1}{4}\left[\frac{(x-y)^{2}}{s}+s(x+y)^{2}\right]}}{s^{\nu+\frac{3}{2}}} d s \lesssim C(x) \frac{t^{2 \nu} e^{-\frac{y^{2}}{2}}}{(t+|x-y|)^{1+2 \nu}},
$$

where $C(x)=(1+x)^{2 \nu} e^{\frac{x^{2}}{2}}$.

PRoof: We shall crudely enlarge the integral in (5.34) to the range $\int_{0}^{1 / 3}$. This last integral was already estimated in [5] and [4], by a similar procedure to the one used in the last subsection. More precisely, from the estimates in [4, Lemma 3.2], formula (3.16), it follows that

$$
t^{2 \nu} \int_{0}^{\frac{1}{2}} \frac{e^{-\frac{c t^{2}}{s}-\frac{1}{4}\left[\frac{(x-y)^{2}}{s}+s(x+y)^{2}\right]}}{s^{\nu+\frac{3}{2}}} d s \lesssim \frac{t^{2 \nu}(1+x)^{2 \nu} e^{\frac{x^{2}-y^{2}}{2}}}{(t+|x-y|)^{1+2 \nu}},
$$

which agrees with (5.34).

5.6. Proof of Propositions 5.1 and $\mathbf{5 . 2}$. Proposition 5.2 follows by putting together Lemmas 5.5, 5.7, 5.8 and 5.10. Concerning Proposition 5.1, the lower bound was shown in Lemma [5.4, while the upper bound also follows from Lemmas 5.5, 5.7 and 5.8, at least when $y<\frac{x}{2}$ or $y>M x$. This actually implies the asserted result for all $x$ and $y$, since when $y$ belongs to the compact set $\left[\frac{x}{2}, M x\right]$, the continuous function $y \rightarrow P_{t}(x, y) / \Phi(y)$ is bounded above by a constant $c_{2}(t, x)$.

5.7. Proof of Corollary [5.3. By Proposition 5.2, observe that

$$
P_{t} f(x) \lesssim \frac{C_{1}(x)}{t} \int_{\mathbb{R}_{+}} \frac{g(y) d y}{\left(1+\frac{|x-y|}{t}\right)^{1+2 \nu}}+C_{2}(x)\left(1 \vee t_{0}\right)^{2 \nu}\|f\|_{L^{1}(\Phi)},
$$

where $g(y)=f(y) e^{-\frac{y^{2}}{2}} \chi_{\left\{\frac{x}{2}<y<M x\right\}}$. The first term is then controlled by a maximal function by a standard slicing argument.

Remark 5.11. We wrote in (1.9) a different version of (5.10) with $\mathcal{M}^{\text {loc }}(f \Phi)$ in place of $\mathcal{M}^{\mathrm{loc}}\left(f e^{-\frac{y^{2}}{2}}\right)$. Since $x \approx y$,

$$
\mathcal{M}^{\operatorname{loc}}(f \Phi)(x) \approx \frac{\langle x\rangle^{\alpha+\frac{1}{2}}}{[\log (e+x)]^{1+\nu}(1+x)^{\mu+\frac{1}{2}}} \mathcal{M}^{\operatorname{loc}}\left(f e^{-\frac{y^{2}}{2}}\right)(x),
$$


so they are actually equivalent modulo $x$-constants. The write-up in (1.9) has the advantage of remaining valid for other Laguerre systems; see \$7 below.

\section{Proofs}

As indicated in $\S 1$ we present the proof of Theorems 1.1 and 1.2 for the differential operator $L$ in (5.1) and the function $\Phi$ in (5.6). We postpone to 97 the proof of the results for the other systems mentioned in Table 1.

6.1. Proof of Theorem 1.1. First of all, it is an immediate consequence of Proposition 5.1 that $P_{t}|f|(x)<\infty$ for some (or all) $t, x>0$ if and only if $f \in L^{1}(\Phi)$. This justifies that $f \in L^{1}(\Phi)$ is the right setting for this problem. Notice also that taking derivatives of the kernel $P_{t}(x, y)$ in (5.5) with respect to $t$ does not worsen its decay in $y$, so $P_{t} f(x)$ automatically becomes infinitely differentiable in the $t$-variable when $f \in L^{1}(\Phi)$. We can also take as many derivatives as wished with respect to $x$, since the kernel satisfies the pdet

$$
\left[\partial_{x x}-x^{2}-\frac{\alpha^{2}-\frac{1}{4}}{x^{2}}-2 \mu+\partial_{t t}+\frac{1-2 \nu}{t} \partial_{t}\right] P_{t}(x, y)=0
$$

so $x$-derivatives are transformed into $t$-derivatives and do not worsen the decay of $P_{t}(x, y)$ in the $y$-variable. We have thus completed the proof of paragraph (i) and the last statement in Theorem 1.1. We shall now prove a stronger result than (ii), namely that for $f \in L^{1}(\Phi)$

$$
\lim _{t \rightarrow 0^{+}} P_{t} f(x)=f(x), \quad \forall x \in \mathcal{L}_{f}
$$

where $\mathcal{L}_{f}$ denotes the set of Lebesgue points of $f$. When $f(x)=0$ this is easily obtained from the kernel estimates in Proposition 5.2. Indeed,

$$
P_{t} f(x) \lesssim C_{1}(x) \int_{\frac{x}{2}}^{M x} \frac{t^{2 \nu}|f(y)| d y}{(t+|x-y|)^{1+2 \nu}}+C_{2}^{\prime}(x) t^{2 \nu} \int_{\mathbb{R}_{+}}|f| \Phi,
$$

where in the second term we have replaced $(t \vee 1)^{2 \nu}$ by $t^{2 \nu}$ in view of Remarks 5.20 and 5.33 . Thus, this second term vanishes as $t \rightarrow 0$ (actually for all $x \in \mathbb{R}_{+}$). Concerning the first term, it is given by convolution of $\mid f(y) \chi_{\left\{\frac{x}{2}<y<M x\right\}} \in L_{c}^{1}\left(\mathbb{R}_{+}\right)$with a radially decreasing approximate identity, so from well-known results (see e.g. [13, p. 112]), it must vanish as $t \rightarrow 0$ at every Lebesgue point $x$ of $f$ with $f(x)=0$.

It remains to prove (6.1) when $f(x)$ is not necessarily 0 . To show this, we first notice that the first eigenfunction $\varphi=\varphi_{0}^{\alpha}$ (with eigenvalue $\lambda=2(\mu+\alpha+1)$ ) satisfies

$$
P_{t} \varphi=F_{t}(\lambda) \varphi, \quad \text { with } \quad \lim _{t \rightarrow 0} F_{t}(\lambda)=1 .
$$

Indeed, setting $u=t^{2} /(4 v)$ in (1.3), gives

$$
F_{t}(\lambda)=\frac{(t / 2)^{2 \nu}}{\Gamma(\nu)} \int_{0}^{\infty} e^{-\frac{t^{2}}{4 u}-\lambda u} \frac{d u}{u^{1+\nu}}=\frac{1}{\Gamma(\nu)} \int_{0}^{\infty} e^{-v-\frac{t^{2} \lambda}{4 v}} v^{\nu-1} d v \longrightarrow 1, \quad \text { as } t \rightarrow 0 .
$$

Therefore, we can write

$$
P_{t} f(x)-f(x)=P_{t} f(x)-F_{t}(\lambda) f(x)+f(x)\left[F_{t}(\lambda)-1\right]
$$

\footnotetext{
${ }^{\ddagger}$ For a justification that the subordinated integral in (1.3) satifies the pde (1.2), see e.g. [4, §2].
} 
with the last term vanishing as $t \rightarrow 0$. Since $\varphi>0$, the first term can be rewritten as

$$
P_{t} f(x)-\frac{f(x)}{\varphi(x)} P_{t} \varphi(x)=P_{t}\left(f-\frac{f(x)}{\varphi(x)} \varphi\right)(x) .
$$

Setting $g=f-\frac{f(x)}{\varphi(x)} \varphi$, it is easily seen that $g \in L^{1}(\Phi), g(x)=0$ and $x$ is a Lebesgue point of $g$. This last assertion follows from

$$
f_{I_{r}(x)}|g(y)| d y \leq f_{I_{r}(x)}|f(y)-f(x)| d y+\frac{|f(x)|}{\varphi(x)} f_{I_{r}(x)}|\varphi(y)-\varphi(x)| d y,
$$

which vanishes as $r \rightarrow 0$. Thus we can apply our earlier case to $g$ and conclude that $\lim _{t \rightarrow 0} P_{t} g(x)=0$. So the left hand side of (6.2) goes to 0 as $t \rightarrow 0$, establishing (6.1) and completing the proof of Theorem 1.1.

Remark 6.1. A close look at the last part of the proof shows that, when $f \in C([a, b])$ with $[a, b] \Subset \mathbb{R}_{+}$, then the convergence of $P_{t} f(x) \rightarrow f(x)$ is uniform in $x \in[a, b]$.

6.2. Proof of Theorem 1.2. We have to show that $P_{t_{0}}^{*}$ maps $L^{p}(w) \rightarrow L^{p}(v)$, for some weight $v(x)>0$, under the assumption that

$$
\|w\|_{D_{p}(\Phi)}:=\left[\int_{\mathbb{R}_{+}} w^{-\frac{1}{p-1}}(x) \Phi(x)^{p^{\prime}} d x\right]^{1 / p^{\prime}}<\infty
$$

with $\Phi$ defined as in (5.6). We shall use the bound for $P_{t_{0}}^{*}$ in (5.10), namely

$$
\begin{aligned}
P_{t_{0}}^{*} f(x) & \lesssim C_{1}(x) \mathcal{M}_{M}^{\text {loc }}\left(f e^{-\frac{y^{2}}{2}}\right)(x)+C_{2}(x) \int_{\mathbb{R}_{+}}|f(y)| \Phi(y) d y \\
& =I(x)+I I(x),
\end{aligned}
$$

for a suitable $M>1$, and $C_{1}(x), C_{2}(x)$ given explicitly in Proposition 5.2. We first treat the last term, which by Hölder's inequality is bounded by

$$
I I(x) \leq C_{2}(x)\|f\|_{L^{p}(w)}\|w\|_{D_{p}(\Phi)} .
$$

Thus, it suffices to choose a weight $v$ such that $C_{2}(x)=[\log (e+x)]^{1+\nu}(1+x)^{\left|\mu+\frac{1}{2}\right|} e^{\frac{x^{2}}{2}} /\langle x\rangle^{\alpha+\frac{3}{2}}$ belongs to $L^{p}(v)$ to conclude that

$$
\|I I\|_{L^{p}(v)} \leq\left\|C_{2}\right\|_{L^{p}(v)}\|w\|_{D_{p}(\Phi)}\|f\|_{L^{p}(w)} .
$$

For instance we may take any $v(x) \leq v_{2}(x)$ with

$$
v_{2}(x):=\frac{l x^{\left(\alpha+\frac{3}{2}\right) p-1}}{[\log (e / l x)]^{2}} \frac{e^{-\frac{p}{2} x^{2}}}{(1+x)^{N}}
$$

for any $N>1+p\left|\mu+\frac{1}{2}\right|$. We remark that $v_{2} \in D_{q}(\Phi)$ for all $q>p$. Indeed, the local condition was already established in (4.3). For the global condition notice that

$$
\int_{1}^{\infty} v_{2}(x)^{-\frac{q^{\prime}}{q}} \Phi(x)^{q^{\prime}} d x \lesssim \int_{1}^{\infty} e^{-\left(1-\frac{p}{q}\right) \frac{q^{\prime}}{2} x^{2}}(1+x)^{\frac{N q^{\prime}}{q}} d x<\infty .
$$

We now consider the term $I(x)$ in (6.3). We define a new weight $W(x)=w(x) e^{\frac{p}{2} x^{2}}$, and observe that

$$
w \in D_{p}(\Phi) \quad \Longrightarrow \quad W \in D_{p}^{0}\left(\alpha+\frac{1}{2}\right) \cap D_{p}^{\exp }(a), \quad \forall a>0,
$$


for the weight classes defined just before Proposition 3.2. Indeed, the local estimate follows from $\Phi(x) \approx\langle x\rangle^{\alpha+\frac{1}{2}}$ when $x \in(0,1)$, and the global estimate is a consequence of

$$
\|W\|_{D_{p}^{\exp }(a)}=\int_{1}^{\infty} w^{-\frac{p^{\prime}}{p}}(x) e^{-\frac{p^{\prime}}{2} x^{2}} e^{-a p^{\prime} x^{2}} d x \leq C_{a}^{p^{\prime}} \int_{1}^{\infty} w^{-\frac{p^{\prime}}{p}}(x) \Phi(x)^{p^{\prime}} d x,
$$

with $C_{a}=\max _{x \geq 1}|\log (e+x)|^{1+\nu}|1+x|^{\mu+\frac{1}{2}} e^{-a x^{2}}<\infty$.

We shall now set

$$
v_{1, \varepsilon}(x)=\frac{e^{-\frac{p x^{2}}{2}}}{(1+x)^{2 p \nu}} \mathscr{V}(x) \rho_{\varepsilon}(\mathscr{V}(x)), \quad \text { where } \mathscr{V}(x)=\left[\mathcal{M}_{M}^{\text {loc }}\left(w^{-\frac{p^{\prime}}{p}} e^{-\frac{p^{\prime} x^{2}}{2}}\right)(x)\right]^{-\frac{p}{p^{\prime}}}
$$

(or $v_{1, \varepsilon}(x)=(1+x)^{-2 p \nu} e^{-\frac{p x^{2}}{2}} V_{\varepsilon}(x)$ in the notation of (3.1)). Given $f \in L^{p}(w)$, we denote $\tilde{f}(y)=f(y) e^{-\frac{y^{2}}{2}}$ which is a function in $L^{p}(W)$. Then, using the two-weight inequality for $\mathcal{M}_{M}^{\text {loc }}$ in Theorem 3.1, and the expression for $C_{1}(x)=(1+x)^{2 \nu} e^{\frac{x^{2}}{2}}$, we see that, for any $v \leq v_{1, \varepsilon}$, the term $I(x)$ in (6.3) is controlled by

$$
\begin{aligned}
\|I(x)\|_{L^{p}(v)}^{p} & \leq \int_{\mathbb{R}_{+}} \frac{C_{1}(x)^{p} e^{-\frac{p}{2} x^{2}}}{(1+|x|)^{p 2 \nu}}\left|\mathcal{M}_{M}^{\mathrm{loc}} \tilde{f}(x)\right|^{p} V_{\varepsilon}(x) d x \\
& \lesssim\|\tilde{f}\|_{L^{p}(W)}^{p}=\|f\|_{L^{p}(w)}^{p} .
\end{aligned}
$$

So, combining (6.3), (6.4) and (6.9) we have shown that $\left\|P_{t_{0}}^{*} f\right\|_{L^{p}(v)} \lesssim\|f\|_{L^{p}(w)}$, provided

$$
v(x)=\min \left\{v_{1, \varepsilon}(x), v_{2}(x)\right\},
$$

with $v_{1, \varepsilon}(x)$ and $v_{2}(x)$ defined in (6.8) and (6.5).

We only have to verify that, if $q>p$ then we can choose $\varepsilon$ sufficiently small so that $v_{1, \varepsilon} \in D_{q}(\Phi)$ (which implies $v \in D_{q}(\Phi)$ ). This actually follows from (6.6) and Proposition 3.2 Indeed, on the one hand, since $W \in D_{p}^{0}\left(\alpha+\frac{1}{2}\right)$

$$
\int_{0}^{1} v_{1, \varepsilon}(x)^{-\frac{q^{\prime}}{q}} \Phi(x)^{q^{\prime}} d x \lesssim \int_{0}^{1} V_{\varepsilon}(x)^{-\frac{q^{\prime}}{q}} l x^{\left(\alpha+\frac{1}{2}\right) q^{\prime}} d x
$$

which is finite by (i) in the proposition (choosing $\varepsilon$ sufficiently small). On the other hand,

$$
\int_{1}^{\infty} v_{1, \varepsilon}(x)^{-\frac{q^{\prime}}{q}} \Phi(x)^{q^{\prime}} d x \lesssim \int_{1}^{\infty} V_{\varepsilon}(x)^{-\frac{q^{\prime}}{q}} e^{-q^{\prime}\left(1-\frac{p}{q}\right) \frac{x^{2}}{2}}(1+x)^{2 \nu p q^{\prime} / q} d x
$$

and since $W \in D_{p}^{\exp }(a)$ for all $a>0$, we can apply part (ii) of Proposition 3.2 (for a sufficiently small $\varepsilon$ ) to conclude that this is also a finite quantity.

Remark 6.2. Alternative expression for the second weight. A slight modification of the above construction suggests to define a new weight by

$$
v_{\varepsilon}^{\Phi, w}(x):=\min \left\{\Phi(x)^{p}\left[\mathcal{M}^{\mathrm{loc}}\left(w^{-\frac{p^{\prime}}{p}} \Phi^{p^{\prime}}\right)(x)\right]^{-\frac{p}{p^{\prime}}} \Upsilon_{\varepsilon}(x), \frac{l x^{p-1}}{[\log (e / l x)]^{2}} \frac{\Phi(x)^{p}}{(1+x)^{N_{0}}}\right\}
$$

with

$$
\Upsilon_{\varepsilon}(x)=\frac{l x^{\varepsilon N_{1}}}{(1+x)^{N_{2}}} \rho_{\varepsilon}\left(\left[\mathcal{M}^{\mathrm{loc}}\left(w^{-\frac{p^{\prime}}{p}} \Phi^{p^{\prime}}\right)(x)\right]^{-\frac{p}{p^{\prime}}}\right) .
$$


If $N_{0}, N_{1}, N_{2}$ are sufficiently large, then similar arguments as above lead to the boundedness of $P_{t_{0}}^{*}: L^{p}(w) \rightarrow L^{p}\left(v_{\varepsilon}^{\Phi, w}\right)$ for all $\varepsilon>0$, and give also the property that $v_{\varepsilon}^{\Phi, w} \in D_{q}(\Phi)$ if $\varepsilon$ is sufficiently small. We omit the details. The expression in (6.13) has the advantage of remaining valid for the other Laguerre systems in Table 1 (with the corresponding $\Phi$ functions).

\section{Transference to Other LAguerre type Systems}

In this section we show how to transfer the results already proved for the system $\left\{\varphi_{n}^{\alpha}\right\}$ and the operator $L$ to the other Laguerre systems and operators in Table 1 . The procedure is completely general, as one can infer already from the first two cases.

7.1. Results for the system $\psi_{n}^{\alpha}$. The starting point is the identity defining $\psi_{n}^{\alpha}$, namely

$$
\psi_{n}^{\alpha}(y)=a(y) \varphi_{n}^{\alpha}(y), \quad \text { with } a(y)=y^{-\alpha-\frac{1}{2}} .
$$

Clearly, $\varphi_{n}^{\alpha}$ is an eigenvector of $L$ if and only if $\psi_{n}^{\alpha}$ is an eigenvector of the operator

$$
f \longmapsto \Lambda f(x)=a(x) L\left[a^{-1} f\right](x)
$$

(with the same eigenvalue $\lambda_{n}=4 n+2(\alpha+1+\mu)$ ). An elementary computation shows that the differential operator $\Lambda$ obtained in this fashion is exactly the one listed in Table 1 . Remark also that $\left\{\psi_{n}^{\alpha}\right\}$ becomes an orthonormal basis in $L^{2}$ with the measure $a^{-2}(y) d y=$ $y^{2 \alpha+1} d y$.

The identity in (7.1) leads to a pointwise relation of the corresponding heat kernels

$$
e^{-t \Lambda}(x, y)=\sum_{n=0}^{\infty} e^{-\lambda_{n} t} \psi_{n}^{\alpha}(x) \psi_{n}^{\alpha}(y)=a(x) a(y) e^{-t L}(x, y),
$$

and by the subordination formula, also of the corresponding Poisson kernels

$$
P_{t}^{\Lambda}(x, y)=a(x) a(y) P_{t}^{L}(x, y) .
$$

In particular,

$$
P_{t}^{\Lambda} f(x)=\int_{\mathbb{R}_{+}} P_{t}^{\Lambda}(x, y) f(y) a^{-2}(y) d y=a(x) P_{t}^{L}\left[a^{-1} f\right](x) .
$$

From this relation it is clear that Theorem 1.1 becomes true for the operator $\Lambda$ with

$$
\Phi^{\Lambda}(y)=a(y)^{-1} \Phi^{L}(y),
$$

as listed in Table 1. From (7.2) it also follows that $P_{t_{0}}^{*, \Lambda}$ maps $L^{p}(w) \rightarrow L^{p}(v)$ if and only if $P_{t_{0}}^{*, L}$ maps $L^{p}\left(a^{p} w\right) \rightarrow L^{p}\left(a^{p} v\right)$, and hence the necessary and sufficient condition becomes

$$
a^{p} w \in D_{p}\left(\Phi^{L}\right) \Longleftrightarrow\left\|a^{-1} w^{-\frac{1}{p}} \Phi^{L}\right\|_{p^{\prime}}<\infty \Longleftrightarrow w \in D_{p}\left(\Phi^{\Lambda}\right),
$$

as was claimed in Theorem 1.2. For the assertions about the weight $v$ one may argue directly as follows. Observe from (7.2) and Corollary 5.3 that we can write

$$
P_{t_{0}}^{*, \Lambda} f(x) \lesssim C_{1}(x) a(x) \mathcal{M}_{M}^{\text {loc }}\left(f a^{-1} e^{-\frac{y^{2}}{2}}\right)(x)+C_{2}(x) a(x) \int_{\mathbb{R}_{+}}|f| \Phi^{\Lambda},
$$


with a cancellation in the first term due to $a(x) a(y)^{-1} \approx 1$ when $\frac{x}{2}<y<M x$. At this point we can apply the same arguments as in 6.2 . Namely, we construct $v=\min \left\{v_{1, \varepsilon}, v_{2}\right\}$ with the same choice of $v_{1, \varepsilon}$, and with $v_{2}$ in (6.5) now replaced by

$$
v_{2}(x):=\frac{l x^{(2 \alpha+2) p-1}}{[\log (e / l x)]^{2}} \frac{e^{-\frac{p}{2} x^{2}}}{(1+x)^{N}} .
$$

The same proof will give that, for any $q>p$, there is a sufficiently small $\varepsilon$ so that $v \in D_{q}\left(\Phi^{\Lambda}\right)$ (the only difference being that, locally, this condition now becomes $v \in D_{q}^{0}(2 \alpha+1)$ ). We remark that this part will work as well with the choice

$$
v(x)=a^{-p}(x) v^{\Phi^{L}, a^{p} w}(x)=v^{\Phi^{\Lambda}, w}(x),
$$

as defined in (6.13).

7.2. Results for the system $\mathfrak{L}_{n}^{\alpha}$. Consider the following isometry of $L^{2}\left(\mathbb{R}_{+}, d y\right)$

$$
f \longmapsto A f(x)=\sqrt{2 x} f\left(x^{2}\right) .
$$

The systems $\mathfrak{L}_{n}^{\alpha}$ and $\varphi_{n}^{\alpha}$ are related by $\varphi_{n}^{\alpha}=A \mathfrak{L}_{n}^{\alpha}$, or equivalently

$$
\mathfrak{L}_{n}^{\alpha}(y)=\left[A^{-1} \varphi_{n}^{\alpha}\right](y)=(4 y)^{-\frac{1}{4}} \varphi_{n}^{\alpha}(\sqrt{y}) .
$$

In particular, $\mathfrak{L}_{n}^{\alpha}$ is an eigenvector of the operator

$$
\mathfrak{L}=\frac{1}{4} A^{-1} \circ L \circ A,
$$

this time with eigenvalue $\lambda_{n} / 4=n+(\alpha+1+\mu) / 2$. The factor $\frac{1}{4}$ has been added so that $\mathfrak{L}$ coincides with the operator listed in Table 1.

The heat kernels are now related by

$$
e^{-t \mathfrak{L}}(x, y)=\sum_{n=0}^{\infty} e^{-t \lambda_{n} / 4} \mathfrak{L}_{n}^{\alpha}(x) \mathfrak{L}_{n}^{\alpha}(y)=\frac{1}{2(x y)^{\frac{1}{4}}} e^{-\frac{t}{4} L}(\sqrt{x}, \sqrt{y}),
$$

and therefore, a substitution in the subordinated integral in (5.5) gives

$$
P_{t}^{\mathfrak{L}}(x, y)=(16 x y)^{-\frac{1}{4}} P_{t / 2}^{L}(\sqrt{x}, \sqrt{y}) .
$$

Thus, we obtain the formula

$$
P_{t}^{\mathfrak{L}} f(x)=\int_{\mathbb{R}_{+}} P_{t}^{\mathfrak{L}}(x, y) f(y) d y=(4 x)^{-\frac{1}{4}} P_{t / 2}^{L}[A f](\sqrt{x}) .
$$

From this relation one easily deduces Theorem 1.1 for the operator $\mathfrak{L}$, provided that

$$
\Phi^{\mathfrak{L}}(y)=\left[A^{-1} \Phi^{L}\right](y),
$$

which is the function asserted in Table 1 (modulo constants).

To establish the second theorem, first observe from (7.4) and Corollary 5.3 that

$$
P_{t_{0}}^{*, \mathfrak{L}} f(x) \lesssim \frac{C_{1}(\sqrt{x})}{x^{1 / 4}} \mathcal{M}_{M}^{\mathrm{loc}}\left(\sqrt{y} f\left(y^{2}\right) e^{-\frac{y^{2}}{2}}\right)(\sqrt{x})+\frac{C_{2}(\sqrt{x})}{x^{1 / 4}} \int_{\mathbb{R}_{+}} \sqrt{2 y}\left|f\left(y^{2}\right)\right| \Phi^{L}(y) .
$$


We claim that this inequality can be rewritten as

$$
P_{t_{0}}^{*, \mathfrak{L}} f(x) \lesssim C_{1}(\sqrt{x}) \mathcal{M}_{M^{2}}^{\text {loc }}\left(f e^{-\frac{y}{2}}\right)(x)+\frac{C_{2}(\sqrt{x})}{x^{1 / 4}} \int_{\mathbb{R}_{+}}|f(u)| \Phi^{\mathfrak{L}}(u) .
$$

The expression for the second term is clear from (7.5) (after a change of variables $y^{2}=u$ ). To handle the first term, notice that the local region now becomes $\frac{\sqrt{x}}{2}<y<M \sqrt{x}$, which in particular gives $x^{-\frac{1}{4}} \sqrt{y} \approx 1$. We also need the following lemma for the maximal function.

Lemma 7.1. For all $g \geq 0$ and $x \in \mathbb{R}_{+}$,

$$
\mathcal{M}\left(g\left(y^{2}\right) \chi_{\left\{\frac{\sqrt{x}}{2}<y<M \sqrt{x}\right\}}\right)(\sqrt{x}) \lesssim \mathcal{M}\left(g(u) \chi_{\left\{\frac{x}{4}<u<M^{2} x\right\}}\right)(x) .
$$

PROOF: This follows essentially from the change of variables $y^{2}=u$,

$$
\begin{aligned}
L H S & \leq \sup _{r>0} \frac{1}{r} \int_{|y-\sqrt{x}|<r} g\left(y^{2}\right) \chi_{\left\{\frac{x}{4}<y^{2}<M^{2} x\right\}} d y \\
& =\sup _{r>0} \frac{1}{r} \int_{|\sqrt{u}-\sqrt{x}|<r} g(u) \chi_{\left\{\frac{x}{4}<u<M^{2} x\right\}} \frac{d u}{2 \sqrt{u}} .
\end{aligned}
$$

In this local range we have $\sqrt{u} \approx \sqrt{x}$, so may take the denominator outside the integral. The local behavior also implies that

$$
|\sqrt{u}-\sqrt{x}|=\left|\int_{x}^{u} \frac{d s}{2 \sqrt{s}}\right| \approx \frac{|u-x|}{\sqrt{x}} .
$$

Therefore, we conclude that

$$
L H S \lesssim \sup _{r>0} \frac{1}{r \sqrt{x}} \int_{|u-x|<r \sqrt{x}} g(u) \chi_{\left\{\frac{x}{4}<u<M^{2} x\right\}} d u \lesssim R H S .
$$

Remark 7.2. A small variation of this proof shows that $x \in \mathcal{L}_{f}$ implies $\sqrt{x} \in \mathcal{L}_{A f}$, so in view of (6.1), the pointwise convergence in (ii) of Theorem 1.1 (for the operator $\mathfrak{L}$ ) actually holds at every Lebesgue point $x$ of $f$.

We have thus shown (7.6). From here one proves Theorem 1.2 (for the operator $\mathfrak{L}$ ) arguing once again as in 86.2 . Remark that, in view of the new constants $C_{1}$ and $C_{2}$ in (77.6), the weight $v=\min \left\{v_{1, \varepsilon}, v_{2}\right\}$ must be defined with

$$
v_{1, \varepsilon}(x)=\frac{e^{-\frac{p x}{2}}}{(1+x)^{p \nu}} \mathscr{V}(x) \rho_{\varepsilon}(\mathscr{V}(x)) \quad \text { and } \quad v_{2}(x)=\frac{l x^{\left(\frac{\alpha}{2}+1\right) p-1}}{[\log (e / l x)]^{2}} \frac{e^{-\frac{p}{2} x}}{(1+x)^{N}}
$$

where $\mathscr{V}(x)=\left[\mathcal{M}_{M^{2}}^{\text {loc }}\left(w^{\frac{1}{1-p}} e^{-\frac{p^{\prime} x}{2}}\right)(x)\right]^{1-p}$ and $N>1+\frac{p}{2}\left(\left|\mu+\frac{1}{2}\right|-\frac{1}{2}\right)$. Then, the same proof as before gives that $P_{t_{0}}^{*}: L^{p}(w) \rightarrow L^{p}(v)$ if $w \in D_{p}\left(\Phi^{\mathfrak{L}}\right)$. One can also establish (with a few obvious modifications) that for every given $q>p$, there is a sufficiently small $\varepsilon$ so that $v \in D_{q}\left(\Phi^{\mathfrak{L}}\right)$. Once again, we may also replace this weight by $v^{\Phi^{\mathfrak{L}}, w}(x)$, as defined in (6.13). 
7.3. Results for the system $\ell_{n}^{\alpha}$. Remember that these functions satisfy

$$
\ell_{n}^{\alpha}(y)=a(y) \mathfrak{L}_{n}^{\alpha}(y), \quad \text { with } a(y)=y^{-\frac{\alpha}{2}} .
$$

Thus, they are eigenvectors of the differential operator

$$
f \mapsto \mathscr{L} f(x)=a(x) \mathfrak{L}\left[a^{-1} f\right](x)
$$

(with the same eigenvalues as $\mathfrak{L}_{n}^{\alpha}$ ) and constitute an orthonormal system in $L^{2}\left(a(y)^{-2} d y\right.$ ). One then derives Theorems 1.1 and 1.2 for the operator $\mathscr{L}$, from the known results about $\mathfrak{L}$, by repeating exactly the same arguments that we gave in $\$ 7.1$. We leave the details to the reader.

7.4. Results for the Laguerre polynomials $L_{n}^{\alpha}$. This system and the corresponding operator $\mathbb{L}$ in (1.1) are the ones considered in the statements of $\$$, so we shall give a few more details here. First of all, recall that $L_{n}^{\alpha}$ and $\mathfrak{L}_{n}^{\alpha}$ are linked by

$$
L_{n}^{\alpha}(y)=a(y) \mathfrak{L}_{n}^{\alpha}(y), \quad \text { with } a(y)=y^{-\frac{\alpha}{2}} e^{y / 2} .
$$

Thus, the functions $L_{n}^{\alpha}$ are orthonormal in $L^{2}\left(a(y)^{-2} d y\right)=L^{2}\left(y^{\alpha} e^{-y} d y\right)$, and are also eigenvectors of the differential operator

$$
f \mapsto \mathbb{L} f(x)=a(x) \mathfrak{L}\left[a^{-1} f\right](x),
$$

with the same eigenvalues as $\mathfrak{L}_{n}^{\alpha}$, namely $n+(\alpha+1+\mu) / 2$. We remark that $\mathbb{L}$ coincides with the operator defined in (1.1) when we set $m=(\alpha+1+\mu) / 2$. Thus, the heat and Poisson kernels of these two operators are related by

$$
e^{-t \mathbb{L}}(x, y)=\sum_{n=0}^{\infty} e^{-(n+m) t} L_{n}^{\alpha}(x) L_{n}^{\alpha}(y)=a(x) a(y) e^{-t \mathfrak{L}}(x, y),
$$

and

$$
P_{t}^{\mathbb{L}}(x, y)=a(x) a(y) P_{t}^{\mathfrak{L}}(x, y) .
$$

This implies the identity

$$
P_{t}^{\mathbb{L}} f(x)=\int_{\mathbb{R}_{+}} P_{t}^{\mathbb{L}}(x, y) f(y) y^{\alpha} e^{-y} d y=a(x) P_{t}^{\mathfrak{L}}\left[f y^{\frac{\alpha}{2}} e^{-\frac{y}{2}}\right](x),
$$

from which one deduces the validity of Theorem 1.1 for the operator $\mathbb{L}$, provided

$$
\Phi^{\mathbb{L}}(x)=y^{\frac{\alpha}{2}} e^{-\frac{y}{2}} \Phi^{\mathfrak{L}}(y)=\frac{y^{\alpha} e^{-y}}{(1+y)^{\frac{1+\alpha+\mu}{2}}[\log (e+x)]^{1+\nu}} .
$$

Note that this coincides with the function in (1.4) since we have set $m=(\alpha+1+\mu) / 2$.

Moreover, (7.9) combined with (7.6) implies the estimate

$$
P_{t_{0}}^{*, \mathbb{L}} f(x) \lesssim C_{1}^{\mathbb{L}}(x) \mathcal{M}_{M^{2}}^{\text {loc }}\left(f e^{-y}\right)(x)+C_{2}^{\mathbb{L}}(x) \int_{\mathbb{R}_{+}}|f(u)| \Phi^{\mathbb{L}}(u)=I(x)+I I(x),
$$

with the new constants

$$
C_{1}^{\mathbb{L}}(x)=(1+x)^{\nu} e^{x} \quad \text { and } \quad C_{2}^{\mathbb{L}}(x)=(1+x)^{\left(\left|\mu+\frac{1}{2}\right|-\alpha-\frac{1}{2}\right) / 2} e^{x} / l x^{\alpha+1} .
$$


We now apply the same arguments as in $\lcm{6.2}$ to show that, for a suitable weight $v$, we have $\left\|P_{t_{0}}^{*, \mathbb{L}} f\right\|_{L^{p}(v)} \lesssim\|f\|_{L^{p}(w)}$, under the assumption $w \in D_{p}\left(\Phi^{\mathbb{L}}\right)$. Indeed, to control the second term $I I(x)$ we choose a weight $v_{2}$ such that $C_{2}^{\mathbb{L}} \in L^{p}\left(v_{2}\right)$, namely

$$
v_{2}(x)=\frac{l x^{(\alpha+1) p-1}}{[\log (e / l x)]^{2}} \frac{e^{-p x}}{(1+x)^{N}},
$$

with say $N>1+p\left(m+\left|\alpha+\frac{1}{2}\right|\right)$. It is not difficult to verify that $v_{2} \in D_{q}\left(\Phi^{\mathbb{L}}\right)$ for all $q>p$. To control the first term we set

$$
v_{1, \varepsilon}(x)=\frac{e^{-p x}}{(1+x)^{p \nu}} \mathscr{V}(x) \rho_{\varepsilon}(\mathscr{V}(x)) \text { with } \mathscr{V}(x)=\left[\mathcal{M}_{M^{2}}^{\text {loc }}\left(w^{\frac{1}{1-p}} e^{-p^{\prime} x}\right)(x)\right]^{1-p} .
$$

That is, if $W(x)=w(x) e^{p x}$, then $v_{1, \varepsilon}(x)=(1+x)^{-p \nu} e^{-p x} V_{\varepsilon}(x)$ with the notation in (3.1). So we may quote Theorem 3.1 to obtain

$$
\|I\|_{L^{p}\left(v_{1, \varepsilon}\right)} \lesssim\left[\int_{\mathbb{R}_{+}}\left|\mathcal{M}_{M^{2}}^{\mathrm{loc}}\left(f e^{-y}\right)(x)\right|^{p} V_{\varepsilon}(x) d x\right]^{\frac{1}{p}} \lesssim\left\|f e^{-y}\right\|_{L^{p}(W)}=\|f\|_{L^{p}(w)} .
$$

Again, it is not difficult to verify that for a sufficiently small $\varepsilon$ one has $v_{1, \varepsilon} \in D_{q}\left(\Phi^{\mathbb{L}}\right)$, arguing as in the last part of 86.2 . Thus, Theorem 1.2 holds with $v=\min \left\{v_{1, \varepsilon}, v_{2}\right\}$. Alternatively, with the notation in (6.13), one may as well choose the weight $v^{\Phi^{\mathbb{L}}, w}(x)$.

Acknowledgements: We wish to thank José Luis Torrea for many useful conversations around these topics at the earlier stages of this work. First and third authors partially supported by grants MTM2013-40945-P, MTM2013-42220-P and MTM2014-57838-C2-1-P from MINECO (Spain), and grants 19368/PI/14 and 19378/PI/14 from Fundación Séneca, (Región de Murcia, Spain). Second and fourth authors partially supported by grants from Consejo Nacional de Investigaciones Científicas y Técnicas (CONICET) and Universidad Nacional del Litoral (Argentina).

\section{REFERENCES}

[1] L. Carleson And P. Jones, Weighted norm inequalities and a theorem of Koosis. Mittag-Leffler Inst., report n. 2, 1981.

[2] A. Chicco Ruiz and E. Harboure, Weighted norm inequalities for heat-diffusion Laguerres semigroups. Math. Z. 257 (2007), 329-354.

[3] J. García-Cuerva and J.L. Rubio de Francia, Weighted norm inequalities and related topics. North-Holland Publishing Co., Amsterdam, 1985.

[4] G. Garrigós, A weak 2-weight problem for the Poisson-Hermite semigroup. To appear in Proc VI CIDAMA Conference, Antequera (Spain), Sept 2014.

[5] G. Garrigós, S. Hartzstein, T. Signes, J.L. Torrea, B. Viviani, Pointwise convergence to initial data of heat and Laplace equations. To appear in Trans. Amer. Math. Soc.

[6] S. I. Hartzstein, J. L. Torrea and B. E. Viviani, A note on the convergence to initial data of Heat and Poisson equation. Proc. Amer. Math. Soc. 141 (2013), 1323-1333.

[7] L. Liu ANd P. SJögren, On the global Gaussian Lipschitz space. Preprint 2015, available in arxiv.

[8] B. Muckenhoupt, Poisson integrals for Hermite and Laguerre expansions. Trans. Amer. Math. Soc. 139 (1969), 231-242.

\footnotetext{
${ }^{\S}$ With the quadratic exponentials $e^{x^{2} / 2}$ in 6.7] and (6.12) replaced by linear exponetials $e^{x}$.
} 
[9] A. Nowak, Heat-diffusion and Poisson integrals for Laguerre and special Hermite expansions on weighted $L^{p}$ spaces. Studia Math. 158 (3) (2003), 239-268.

[10] A. Nowak And K. Stempak, Weighted estimates for the Hankel transform transplantation operator. Tohoku Math. J. 58 (2) (2006), 277-301.

[11] J.L. Rubio De Francia, Weighted norm inequalities and vector valued inequalities. In Harmonic analysis (Minneapolis 1981), Lecture Notes Math. 908, Springer 1982, 86-101.

[12] K. Stempak, Heat diffusion and Poisson integrals for Laguerre expansions. Tohoku Math. J. 46 (1994), 83-104.

[13] E. Stein and R. Shakarchi, Real Analysis. Princeton University Press 2005.

[14] P. Stinga, J.L. Torrea, Extension problem and Harnack's inequality for some fractional operators. Comm. Partial Differential Equations 35 (11) (2010), 2092-2122.

Garrigós and Signes, Departamento de Matemáticas, Universidad de Murcia, 30100 Murcia, SPAIN. Email: gustavo.garrigos@um.es, tmsignes@um.es

Hartzstein and Viviani, imAL (UNL-CONiCET) y FiQ (Universidad NACiOnal del Litoral), CCt Conicet Santa Fe Colectora Ruta Nac. N168, Paraje El Pozo, 3000 Santa Fe, ArGENTINA. Email: shartzstein@santafe-conicet.gov.ar, viviani@santafe-conicet.gov.ar 